\title{
COMPUTING QUADRATIC FUNCTION FIELDS WITH HIGH 3-RANK VIA CUBIC FIELD TABULATION
}

\author{
P. ROZENHART, M.J. JACOBSON, JR. AND R. SCHEIDLER
}

\begin{abstract}
In this paper, we present extensive numerical data on quadratic function fields with non-zero 3-rank. We use a function field adaptation of a method due to Belabas for finding quadratic number fields of high 3-rank. Our algorithm relies on previous work for tabulating cubic function fields of bounded discriminant [28] but includes a significant novel improvement when the discriminants are imaginary. We provide numerical data for discriminant degree up to 11 over the finite fields $\mathbb{F}_{5}, \mathbb{F}_{7}, \mathbb{F}_{11}$ and $\mathbb{F}_{13}$. In addition to presenting new examples of fields of minimal discriminant degree with a given 3-rank, we compare our data with a variety of heuristics on the density of such fields with a given 3 -rank, which in most cases supports their validity.
\end{abstract}

1. Introduction. Let $D$ be a square-free non-constant polynomial in $\mathbb{F}_{q}[t]$ and $\mathrm{Cl}(D)$ the ideal class group of the quadratic function field $\mathbb{F}_{q}(t, \sqrt{D})$. For any prime $\ell$, the number $r_{\ell}(D)$, which denotes the number of cyclic factors in the $\ell$-Sylow subgroup of $\mathrm{Cl}(D)$, is called the $\ell$-rank of the group $\mathrm{Cl}(D)$. In short, we say that the quadratic function field $\mathbb{F}_{q}(t, \sqrt{D})$ has $\ell$-rank $r$ if $\mathrm{Cl}(D)$ has $\ell$-rank equal to $r$.

In this paper, we present extensive numerical data on quadratic function fields of discriminant $D$ with bounded degree (where $-3 D$ is imaginary or unusual) and non-zero 3-rank. Our algorithm is an adaptation of our previous work [28] for tabulating cubic function fields of bounded discriminant degree and is inspired by Belabas' algorithm [5] for tabulating quadratic number fields of bounded discriminant with 3-rank greater than zero. The rapid tabulation of all cubic function fields with bounded discriminant degree is key to this approach; alternative meth-

2010 AMS Mathematics subject classification. Primary 11R11, 11R58, 11R65, $11 \mathrm{Y} 40$.

Keywords and phrases. Quadratic function field, ideal class group, three rank.

The second and third authors' research is supported by NSERC of Canada.

Received by the editors on July 16, 2013, and in revised form on January 26, 2014. 
ods such as those of $[\mathbf{1 9}, \mathbf{2 6}, \mathbf{3 3}]$ that find all non-isomorphic cubic function fields of a single given discriminant are less efficient for this purpose (because they require the computation of the ideal class group of the associated quadratic function field) and are thus not suitable.

While the basic ideas of this paper stem from the first author's doctoral dissertation [27, Chapter 7], written under the supervision of the last two authors, a large portion of the material herein is new and represents a significant improvement to [27]. Our contributions are as follows. First, our basic algorithm (Algorithm 3.1) functions in much the same way as the tabulation algorithm from [27] (appearing in [28]), essentially looping over the four possible coefficients of associated binary cubic forms. In the imaginary case, we improve on this method by making use of the $\mathbb{F}_{q}(t)$-automorphisms that send $t$ to $t+\alpha$ with $\alpha \in \mathbb{F}_{q}^{*}$; this new improvement is unique to the function field setting, and our analysis shows that it results in a speed up by a factor of approximately $q$ in most cases over the basic version of our algorithm. These improvements allowed us to push our computations beyond those in [27] and also explain why the number of fields with a given positive 3-rank have certain divisibility properties observed in [27].

Our 3-rank algorithm, like Belabas's [5], is exhaustive in the sense that all quadratic discriminants of any fixed degree that define quadratic function fields with positive 3-rank are produced by the method. As a consequence, the resulting algorithm also produces minimum discriminant sizes for any given positive 3 -rank value. This is in contrast to $[4,6]$, which present methods for finding quadratic function fields with high 3-rank whose discriminants are small, but not necessarily minimal. Our second contribution is the identification of these minimal discriminant sizes for a given positive 3 -rank value $r$, rather than record 3-rank values.

Our third contribution is that, since our method is exhaustive, we can generate data on the distribution of 3-rank values up to a fixed bound $B$ on $\operatorname{deg}(D)$. Cohen and Lenstra $[\mathbf{8}, \mathbf{9}]$ gave heuristics on the behavior of class groups of quadratic number fields. For example, they provided heuristic estimates for the probability that the $\ell$-rank of a given class group is equal to $r$ for a given prime $\ell$ and non-negative integer $r$. None of these heuristics are proved, but there is a large amount of numerical evidence supporting their validity, as seen, for example, in $[\mathbf{2 0}, \mathbf{3 1}]$. These heuristics imply that the ideal class group 
of a quadratic number field is expected to have low $\ell$-rank for any prime $\ell$. Consequently, there is a large body of literature devoted to the construction of families of quadratic number fields of large $\ell$-rank, with 3-ranks of particular interest.

The function field analogue of the Cohen-Lenstra heuristics, the Friedman-Washington heuristics [14], attempt to explain statistical observations about divisor class groups of quadratic function fields. Some progress has been made in trying to prove these heuristics, most notably by Achter [1, 2], Ellenberg, Venkatesh and Westerland [12, 32] and Garton [16]. These results are somewhat weaker than the original heuristics, as they rely on the size $q$ of the underlying finite field tending to infinity in addition to the genus of the function field. Previous results attempting to numerically verify the Friedman-Washington heuristics include computations of class groups for small genus over small base fields by Feng and Sun [13] and computations of class groups of real quadratic function fields of genus 1 over large base fields by Friesen [15]. To the knowledge of the authors the computational data contained herein is the most extensive since the work of Feng and Sun [13] and Friesen [15].

We generated examples of minimal genus and 3-rank values as large as four for quadratic function fields over $\mathbb{F}_{q}$ for $q=5,7,11,13$. As expected, we did not find fields with higher 3-rank than any known examples, but we did find numerous examples of fields with 3-rank as high as four and smaller genus than any others known. In addition, the data we generated yields evidence for the validity of the FriedmanWashington heuristic for $q=5,11$. Due to the presence of cube roots of unity, the data for $q=7,13$ does not agree closely with Friedman-Washington and Ellenberg, et al., but instead with some recent conjectures of Malle $[\mathbf{2 4}, \mathbf{2 5}]$ for number fields, and a new distribution result for function fields of Garton [16, 17]. Our data suggests that all the doubly-asymptotic results of Ellenberg, et al., and Garton, where $q \rightarrow \infty$ as well as $g \rightarrow \infty$, may also hold for fixed $q$.

This paper is organized as follows. After a brief review of some preliminaries from the theory of algebraic function fields and cubic function field tabulation in Section 2, we proceed with a short discussion of the basic algorithm in Section 3 and the improved algorithm for imaginary discriminants in Section 4. The algorithm's complexity is analyzed in Section 5. We discuss the Friedman-Washington heuristics 
and related results in Section 6. The 3-rank data generated is presented in Section 7, and a comparison to the Friedman-Washington heuristics, the Achter and Ellenberg, et al., distribution, Malle's conjectured formula and Garton's distribution are presented in Section 8. Finally, we make some concluding remarks and suggest open problems in Section 9 .

2. Preliminaries. In this section, we briefly summarize the main theoretical results underlying our algorithm. None of this material is new (original presentation and proofs can all be found in $[\mathbf{2 7}, \mathbf{2 8}]$ ), but we nevertheless include this summary in order to make our presentation more self-contained.

Let $\mathbb{F}_{q}$ be a finite field of characteristic at least 5 , and set $\mathbb{F}_{q}^{*}=$ $\mathbb{F}_{q} \backslash\{0\}$. Denote by $\mathbb{F}_{q}[t]$ and $\mathbb{F}_{q}(t)$ the ring of polynomials and the field of rational functions in the variable $t$ over $\mathbb{F}_{q}$, respectively. For any non-zero $H \in \mathbb{F}_{q}[t]$ of degree $n=\operatorname{deg}(H)$, we let $|H|=q^{n}=q^{\operatorname{deg}(H)}$ and denote by $\operatorname{sgn}(H)$ the leading coefficient of $H$. For $H=0$, we set $|H|=0$. This absolute value extends in the obvious way to $\mathbb{F}_{q}(t)$. Note that, in contrast to the absolute value on the rational numbers $\mathbb{Q}$, the absolute value on $\mathbb{F}_{q}(t)$, is non-Archimedean.

A binary quadratic form over $\mathbb{F}_{q}[t]$ is a homogeneous quadratic polynomial in two variables with coefficients in $\mathbb{F}_{q}[t]$. We denote the binary quadratic form $H(x, y)=P x^{2}+Q x y+R y^{2}$ by $H=(P, Q, R)$. The discriminant of $H$ is the polynomial

$$
D(H)=Q^{2}-4 P R \in \mathbb{F}_{q}[t] .
$$

A polynomial $F \in \mathbb{F}_{q}[t]$ is said to be imaginary if $\operatorname{deg}(F)$ is odd, unusual if $\operatorname{deg}(F)$ is even and $\operatorname{sgn}(F)$ is a non-square in $\mathbb{F}_{q}^{*}$, and real if $\operatorname{deg}(F)$ is even and $\operatorname{sgn}(F)$ is a square in $\mathbb{F}_{q}^{*}$. Correspondingly, a binary quadratic form is said to be imaginary, unusual or real according to whether its discriminant is imaginary, unusual or real.

A binary cubic form over $\mathbb{F}_{q}[t]$ is a homogeneous cubic polynomial in two variables with coefficients in $\mathbb{F}_{q}[t]$. We denote the binary cubic form $f(x, y)=a x^{3}+b x^{2} y+c x y^{2}+d y^{3}$ by $f=(a, b, c, d)$. The discriminant of $f=(a, b, c, d)$ is the polynomial

$$
D(f)=18 a b c d+b^{2} c^{2}-4 a c^{3}-4 b^{3} d-27 a^{2} d^{2} \in \mathbb{F}_{q}[t] .
$$


We assume throughout that binary cubic forms $f=(a, b, c, d)$ are primitive, i.e., $\operatorname{gcd}(a, b, c, d)=1$, and irreducible (as a polynomial in $x$ and $y$ ).

Let $F$ be a binary quadratic or cubic form over $\mathbb{F}_{q}[t]$. If $M=\left(\begin{array}{cc}\alpha & \beta \\ \gamma & \delta\end{array}\right)$ is a $2 \times 2$ matrix with entries in $\mathbb{F}_{q}[t]$, then the action of $M$ on $F$ is defined by

$$
(F \circ M)(x, y)=F(\alpha x+\beta y, \gamma x+\delta y) .
$$

We obtain an equivalence relation from this action by restricting to matrices $M \in G L_{2}\left(\mathbb{F}_{q}[t]\right)$, the group of $2 \times 2$ matrices over $\mathbb{F}_{q}[t]$ whose determinant lies in $\mathbb{F}_{q}^{*}$. That is, two binary quadratic or cubic forms $F$ and $G$ over $\mathbb{F}_{q}[t]$ are said to be equivalent if

$$
F(\alpha x+\beta y, \gamma x+\delta y)=G(x, y)
$$

$\alpha \delta-\beta \gamma \in \mathbb{F}_{q}^{*}$. Up to some even power of $\operatorname{det}(M)$, equivalent binary forms have the same discriminant. Furthermore, the action of the group $G L_{2}\left(\mathbb{F}_{q}[t]\right)$ on binary forms over $\mathbb{F}_{q}[t]$ preserves irreducibility and primitivity over $\mathbb{F}_{q}(t)$.

As in the case of integral binary cubic forms, any binary cubic form $f=(a, b, c, d)$ over $\mathbb{F}_{q}[t]$ is closely associated with its Hessian

$$
H_{f}(x, y)=-\frac{1}{4}\left|\begin{array}{cc}
\frac{\partial^{2} f}{\partial x \partial x} & \frac{\partial^{2} f}{\partial x \partial y} \\
\frac{\partial^{2} f}{\partial y \partial x} & \frac{\partial^{2} f}{\partial y \partial y}
\end{array}\right|=(P, Q, R),
$$

where $P=b^{2}-3 a c, Q=b c-9 a d$ and $R=c^{2}-3 b d$. Note that $H_{f}$ is a binary quadratic form over $\mathbb{F}_{q}[t]$. The Hessian has a number of useful properties, which are easily verified by direct computation:

$$
\begin{gathered}
H_{f \circ M}=(\operatorname{det} M)^{2}\left(H_{f} \circ M\right) \text { for any } M \in G L_{2}\left(\mathbb{F}_{q}[t]\right), \quad \text { and } \\
D\left(H_{f}\right)=-3 D(f) .
\end{gathered}
$$

We now briefly summarize the reduction theory for binary quadratic and cubic forms. Fix a primitive root $h$ of $\mathbb{F}_{q}^{*}$. As in Artin [3], we only consider quadratic discriminants $D$ endowed with the normalization $\operatorname{sgn}(D)=1$ or $\operatorname{sgn}(D)=h$, where 1 or $h$ is chosen depending on whether or not $\operatorname{sgn}(D)$ is a square in $\mathbb{F}_{q}^{*}$. We can impose this restriction since the discriminant of a function field is only unique up to square factors 
in $\mathbb{F}_{q}^{*}$. Define the set

$$
S=\left\{h^{i}: 0 \leq i \leq(q-3) / 2\right\}
$$

Then $a \in S$ if and only if $-a \notin S$.

Definition 2.1. (Summary of Definitions 3.1, 3.5, and 4.1 of [28]).

(1) Let $H=(P, Q, R)$ be an imaginary or unusual binary quadratic form of discriminant $D$. Then $H$ is reduced if

(a) $|Q|<|P|$, and either $Q=0$ or $\operatorname{sgn}(Q) \in S$;

(b) Either $|P|<|R|$ and $\operatorname{sgn}(P) \in\{1, h\}$, or $|P|=|R|$ and $\operatorname{sgn}(P)=1$;

(c) When $|P|=|R|$ and $\operatorname{sgn}(P)=1$, then $f$ is lexicographically smallest among the $q+1$ partially reduced binary quadratic forms in its equivalence class satisfying conditions (a) and (b) above.

(2) Let $f=(a, b, c, d)$ be a binary cubic form with imaginary or unusual Hessian $H_{f}=(P, Q, R)$ of discriminant $-3 D$. Then $f$ is reduced if (a) $\operatorname{sgn}(a) \in S$, and, if $Q=0$, then $\operatorname{sgn}(d) \in S$.

(b) $H_{f}$ is reduced, and, in addition, if $|P|=|R|$, then $f$ is lexicographically smallest among all binary cubic forms in its equivalence class with Hessian $H_{f}$.

The proof of the following theorem can be found in [28, Theorems 3.6, 4.2].

\section{Theorem 2.2.}

(i) Every equivalence class of imaginary or unusual binary quadratic forms contains a unique reduced representative, and there are only finitely many reduced imaginary or unusual binary quadratic forms of any given discriminant.

(ii) Every equivalence class of binary cubic forms with imaginary or unusual Hessian contains a unique reduced representative, and there are only finitely many reduced binary cubic forms of any given discriminant with imaginary or unusual Hessian. 
Recall from [28, Theorem 5.3] that, if $f=(a, b, c, d)$ is a reduced binary cubic form of discriminant $D$, then

$$
\begin{aligned}
\operatorname{deg}(a), \operatorname{deg}(b) & \leq \operatorname{deg}(D) / 4, \\
\operatorname{deg}(a c), \operatorname{deg}(b c), \operatorname{deg}(a d) & \leq \operatorname{deg}(D) / 2 .
\end{aligned}
$$

The tabulation of $\mathbb{F}_{q}(t)$-isomorphism classes of cubic function fields as performed in $[\mathbf{2 7}, \mathbf{2 8}, \mathbf{2 9}]$ used the Davenport-Heilbronn bijection between $\mathbb{F}_{q}(t)$-isomorphism classes of cubic function fields and a certain collection $\mathcal{U}$ of $G L_{2}\left(\mathbb{F}_{q}[t]\right)$-isomorphism classes of binary cubic forms. This set $\mathcal{U}$ includes all classes of primitive, irreducible binary cubic forms with square-free discriminant, which is all that is required in our context. The Davenport Heilbronn correspondence simply assigns each form $f(x, y)$ in $\mathcal{U}$ the irreducible cubic polynomial $f(x, 1)$.

In analogy to the number field terminology, a polynomial in $\mathbb{F}_{q}[t]$ is said to be a fundamental discriminant if it is square-free, of degree at least 3 , and has leading coefficient 1 or $h$. In order to compute the 3-rank of a quadratic function field of square-free discriminant $D$, we need only count the number of $\mathbb{F}_{q}(t)$-isomorphism classes of cubic function fields of that same discriminant and with at least two infinite places, see Theorem 2.3 below. In turn, to list all $\mathbb{F}_{q}(t)$ isomorphism classes of cubic function fields of discriminant $D$, it suffices to enumerate the corresponding unique reduced irreducible binary cubic forms. To generate all the desired quadratic function fields up to a given discriminant degree bound $B$, we employ the degree bounds (2.1) (with $\operatorname{deg}(D)$ replaced by $B$ ) in nested loops over the coefficients of a binary cubic form. For each such form, we check whether it is reduced and has a discriminant $D$ so that $-3 D$ is a fundamental imaginary or unusual discriminant of degree at most $B$. A more precise description of the algorithm is given in Section 3 .

Our algorithm for generating quadratic function fields of positive 3rank relies on key connections between quadratic and cubic fields of the same discriminant. A modified version of a theorem of Hasse [18] for the function field setting appears in [19] and gives a precise formula for the number of isomorphism classes of cubic function fields for a fixed square-free discriminant $D$ in terms of the 3-rank of the quadratic field of discriminant $D$. Specifically, if $D$ is a square-free polynomial in $\mathbb{F}_{q}[t]$ and $K=\mathbb{F}_{q}(t, \sqrt{D})$, then the number of $\mathbb{F}_{q}(t)$-isomorphism classes of cubic function fields of discriminant $D$ with at least two infinite places 
is

$$
\left(3^{r_{3}(D)}-1\right) / 2
$$

where $r_{3}(D)$ is the 3-rank of the ideal class group of the quadratic function field $K$.

Note that the Hasse count omits the classes of cubic function fields with just one infinite place. To include these classes, we require a more refined count. Let $n$ be any non-square in $\mathbb{F}_{q}$, and suppose that $D$ is unusual. Then the real discriminant $D^{\prime}=n D$ is said to be the dual of $D$, and the real quadratic function field $K^{\prime}=\mathbb{F}_{q}\left(t, \sqrt{D^{\prime}}\right)$ is the dual of the unusual quadratic function field $K=\mathbb{F}_{q}(t, \sqrt{D})$. Let $\ell$ be an odd prime dividing $q+1$. If $r$ and $r^{\prime}$ denote the $\ell$-rank of $K / \mathbb{F}_{q}(t)$ and $K^{\prime} / \mathbb{F}_{q}(t)$, respectively, then $r=r^{\prime}$ or $r=r^{\prime}+1$. In the latter case, the regulator of $K^{\prime} / \mathbb{F}_{q}(t)$ is a multiple of $\ell$ (see Lee [23]). The cases $r=r^{\prime}+1$ and $r=r^{\prime}$ are referred to as escalatory and non-escalatory, respectively.

Denote by $\left(e_{1}, f_{1} ; \ldots ; e_{r}, f_{r}\right)$ the signature of the place at infinity of $\mathbb{F}_{q}(t)$ in a finite extension $L$ of $\mathbb{F}_{q}(t)$, so $e_{i}$ is the ramification index and $f_{i}$ the residue degree of the $i$ th infinite place of $L$ for $1 \leq i \leq r$. In the cases of interest, i.e., $-3 D$ imaginary or unusual, we have an exact count of the number of $\mathbb{F}_{q}(t)$-isomorphism classes of cubic function fields of discriminant $D$ and any given signature. The complete statement and proof appear in [19].

Theorem 2.3. Let $D$ in $\mathbb{F}_{q}[t]$ be square-free so that $-3 D$ is imaginary or unusual. Then the number of $\mathbb{F}_{q}(t)$-isomorphism classes of cubic function fields of discriminant $D$ is $\left(3^{r_{3}(-3 D)}-1\right) / 2$. Setting $r=r_{3}(D)$, the possible signatures for these fields and their respective frequencies are as follows:

- If $-3 D$ is imaginary, then all $\left(3^{r}-1\right) / 2$ classes of fields have signature $(1,1 ; 2,1)$.

- If $-3 D$ is unusual and $q \equiv 1(\bmod 3)$, then all $\left(3^{r}-1\right) / 2$ classes of fields have signature $(1,1 ; 1,2)$.

- If $-3 D$ is unusual and $q \equiv-1(\bmod 3)$, then $D$ is the dual discriminant of $-3 D$, and there are two possibilities:

- In the non-escalatory case, all $\left(3^{r}-1\right) / 2$ classes of fields have signature $(1,1 ; 1,1 ; 1,1)$. 
- In the escalatory case, $\left(3^{r}-1\right) / 2$ classes of fields have signature $(1,1 ; 1,1 ; 1,1)$ and the remaining $3^{r}$ such classes have signature $(1,3)$.

\section{Tabulating quadratic function fields with non-zero 3-} rank. We now briefly describe our method for tabulating quadratic function fields of imaginary or unusual fundamental discriminant $-3 D$ with positive 3-rank up to a given bound $B$ on $\operatorname{deg}(D)$. The basic version of our algorithm is a straightforward application of the cubic function field tabulation algorithm of [28]. The main difference is that, instead of outputting minimal polynomials for all fields, we simply increment a counter for each square-free discriminant found. The counter and corresponding discriminant values are then output. Discriminants and the number of cubic fields with that discriminant are stored in a hash table, and output to a file once the main for loops are exited. Specifically, we loop over each coefficient of a binary cubic form satisfying the bounds given in (2.1). For each binary cubic form $f$ encountered in the loop, we test whether or not $f$ is reduced, is squarefree, $-3 D$ is imaginary or unusual and $\operatorname{deg}(D) \leq B$. If this is the case, the number of binary cubic forms found with discriminant $D$ is incremented by one in our hash table. Once the hash table is complete, the value of the counter for each discriminant can then be converted to its proper 3-rank value using Theorem 2.3, depending on which case is appropriate. A modified version of the algorithm, with various improvements including shortening the loop on $d$ as described previously in [28], appears in Algorithm 3.1.

Algorithm 3.1. 3-rank algorithm for computation of quadratic fields where $-3 D$ is imaginary (respectively, unusual).

Input: A prime power $q$ not divisible by 2 or 3 , a primitive root $h$ of $\mathbb{F}_{q}$, the set $S=\left\{1, h, h^{2}, \ldots h^{(q-3) / 2}\right\}$, and a positive integer $B$.

Output: A table where each entry is a square-free discriminant $D$ and a number of the form $\left(3^{r}-1\right) / 2$ with $-3 D$ imaginary (respectively, unusual), $\operatorname{sgn}(-3 D) \in\{1, h\}$ (respectively, $\operatorname{sgn}(-3 D)=h$ ), and $\operatorname{deg}(D) \leq B$. The positive integer $r$ is the 3-rank of the quadratic function field $\mathbb{F}_{q}(t, \sqrt{D})$.

1: for $\operatorname{deg}(a) \leq B / 4 \boldsymbol{A N D} \operatorname{sgn}(a) \in S$ do

2: for $\operatorname{deg}(b) \leq B / 4$ do 


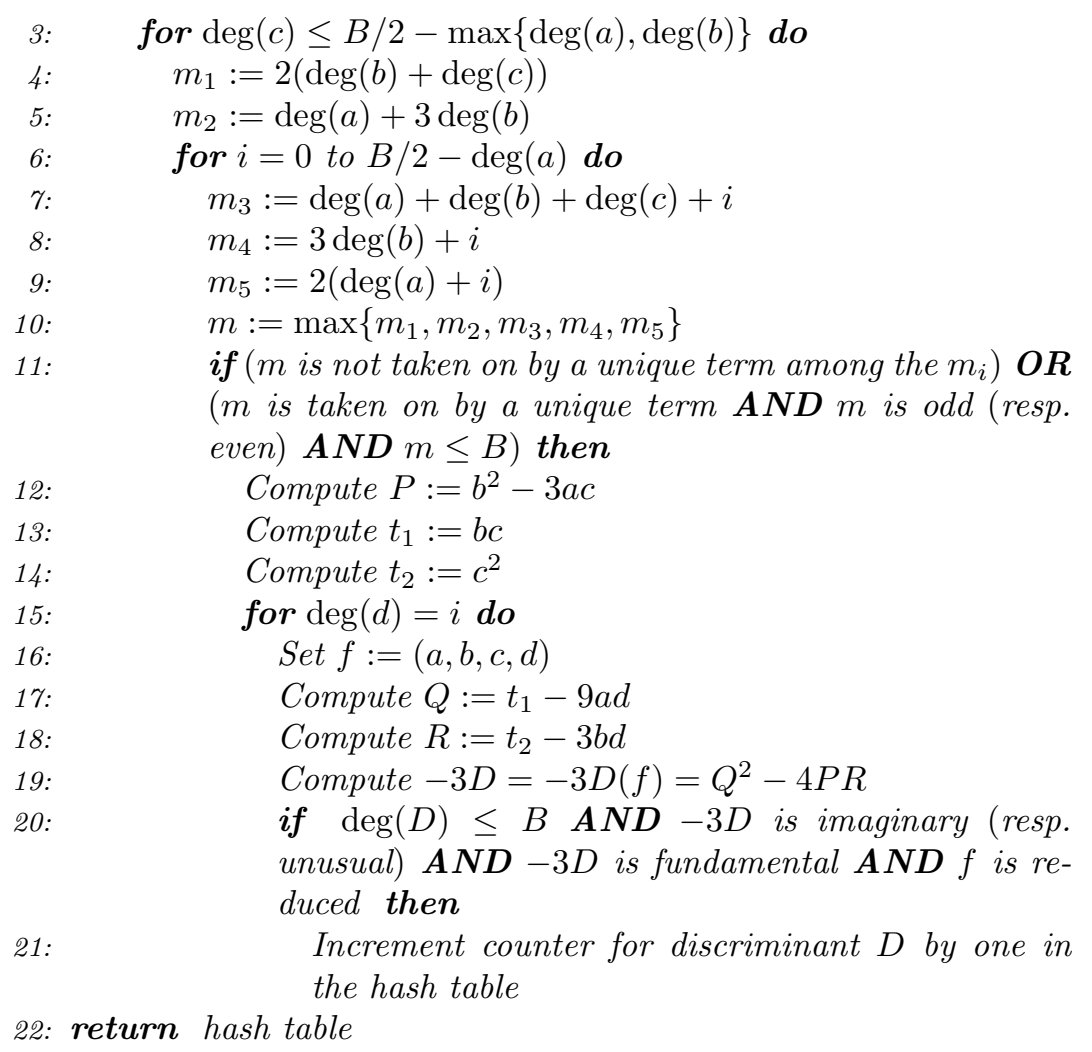

The asymptotic complexity of the algorithm for generating fields with positive 3-rank is the same as for the tabulation algorithm for cubic function fields, namely, $O\left(B^{4} q^{B}\right)$ field operations as $B \rightarrow \infty$ [28], with the $O$-constant cubic in $q$ when $B$ is odd and quartic in $q$ when $B$ is even. The main difference between the two algorithms is that, instead of testing if an equivalence class of binary cubic forms belongs to the Davenport-Heilbronn set, we just need to test whether it has square-free discriminant. This requires only one gcd computation. The 3-rank program then stores the discriminant and the 3-rank data in a hash table, which is output at the end of the algorithm.

In Belabas [5], a number of modifications to the basic algorithm for computing the 3-rank of a quadratic number field were suggested and 
implemented. We give a brief summary of these modifications here and explain why we refrained from making similar changes to our program, since the cost of this is negligible compared to the rest of the algorithm.

First, we did not use Belabas's "cluster" approach. This approach, where one loosens the conditions for a form to be reduced and looks for a large number of forms in a given interval, finally proceeding with class group computations on the clusters found, was not used as we sought to avoid a large number of direct class group computations, except for verification of a small sample of examples. This does, however, warrant further investigation.

The other main variants of Belabas's 3-rank program are dedicated to speeding up the square-free test for integers. As square-free testing for polynomials is straightforward and efficient, this aspect of Belabas's work was not explored.

4. An improved algorithm for $-3 D$ imaginary. In this section, we use certain $\mathbb{F}_{q}(t)$-automorphisms to speed up our algorithm by a factor of $q$ in most cases when $-3 D$ is imaginary. This requires some additional notation. Henceforth, let $p$ denote the characteristic of $\mathbb{F}_{q}$. For any non-constant polynomial $F(t) \in \mathbb{F}_{q}[t]$, let $\operatorname{sgn}_{2}(F)$ denote the coefficient of $t^{\operatorname{deg}(F)-1}$ in $F$ (this is allowed to be zero). We also require a preliminary lemma, which is easily proved by induction on the degree.

Lemma 4.1. Let $F(t) \in \mathbb{F}_{q}[t]$ be a non-zero polynomial. Then $F(t+\beta)=F(t)$ for all $\beta \in \mathbb{F}_{q}$ if and only if $F(t)$ is a polynomial in $t^{q}-t$.

Proof. "Freshmen exponentiation" easily shows that every polynomial in $t^{q}-t$ is invariant under translation by elements in $\mathbb{F}_{q}$. The converse certainly holds for constant polynomials. So, let $F(t) \in \mathbb{F}_{q}[t]$ be non-constant and assume inductively that the converse statement holds for all polynomials of degree less than $\operatorname{deg}(F)$. The constant coefficient of $F(t)$ is $F(0)$, so obviously $t$ divides $F(t)-F(0)$. Replacing $t$ by $t+\beta$ for any $\beta \in \mathbb{F}_{q}$, we see that $t+\beta$ divides $F(t+\beta)-F(0)=F(t)-F(0)$ for all $\beta \in \mathbb{F}_{q}$. It follows that

$$
t^{q}-t=\prod_{\beta \in \mathbb{F}_{q}}(t-\beta)
$$


divides $F(t)-F(0)$. Thus, $F(t)=\left(t^{q}-t\right) G(t)+F(0)$ for some polynomial $G(t) \in \mathbb{F}_{q}[t]$ of degree $\operatorname{deg}(F)-q<\operatorname{deg}(F)$. Now, again by "freshmen exponentiation,"

$$
\begin{aligned}
\left(t^{q}-t\right) G(t)+F(0) & =F(t)=F(t+\beta) \\
& =\left((t+\beta)^{q}-(t+\beta)\right) G(t+\beta)-F(0) \\
& =\left(t^{q}-t\right) G(t+\beta)-F(0),
\end{aligned}
$$

so $G(t+\beta)=G(t)$ for all $\beta \in \mathbb{F}_{q}$. By the induction hypothesis, $G(t)$ is a polynomial in $t^{q}-t$, and hence so is $F(t)$.

The key to our improvements is the following:

Proposition 4.2. For every polynomial $F(t) \in \mathbb{F}_{q}[t]$ whose degree is coprime to $p$, there exists a unique $\beta \in \mathbb{F}_{q}$ such that $\operatorname{sgn}_{2}(F(t+\beta))=0$.

Proof. If $d=\operatorname{deg}(F)$, then $\operatorname{sgn}_{2}(F(t+\beta))=\operatorname{sgn}_{2}(F)+d \beta \operatorname{sgn}(F)$, which vanishes if and only if $\beta=-\operatorname{sgn}_{2}(F) /(d \operatorname{sgn}(F))$.

Corollary 4.3. For every imaginary reduced binary cubic form $f=$ $(a, b, c, d)$ over $\mathbb{F}_{q}[t]$ with imaginary Hessian and $p \nmid \operatorname{deg}(a)$, there exists a unique $\beta \in \mathbb{F}_{q}$ such that $\operatorname{sgn}_{2}(a(t+\beta))=0$ and the form $f_{\beta}=(a(t+\beta), b(t+\beta), c(t+\beta), d(t+\beta))$ is imaginary and reduced.

Proof. The proof follows immediately from Proposition 4.2, since translation does not change the conditions on an imaginary reduced form as specified in Definition 2.1.

Note that, if $f$ has discriminant $D(t)$, then $f_{\beta}$ has discriminant $D(t+\beta)$ which is generally distinct from $D(t)$. If $f$ is a reduced binary cubic form with unusual Hessian, then translating the coefficients by any $\beta$ in $\mathbb{F}_{q}$ produces a partially reduced form, but not necessarily a reduced one. We were unable to find an efficient way to address this problem. We will revisit this issue in Section 9.

The idea for speeding up the 3-rank algorithm is to loop only over polynomials $a$ with $\operatorname{sgn}_{2}(a)=0$ when $\operatorname{deg}(a)$ is not divisible by $p$. Each such $a$ yields $q$ distinct forms

$$
(a(t+\beta), b(t+\beta), c(t+\beta), d(t+\beta))
$$


for each non-zero $\beta \in \mathbb{F}_{q}$ of respective discriminants $D(t+\beta)$, for which the count is appropriately adjusted afterwards. This can be further improved by using the same idea on the polynomials $d$. That is, the algorithm loops only over pairs $(a, d)$ with

$$
p \nmid \operatorname{deg}(a) \text { and } \operatorname{sgn}_{2}(a)=0,
$$

or

$$
p \mid \operatorname{deg}(a), \quad p \nmid \operatorname{deg}(d) \quad \text { and } \quad \operatorname{sgn}_{2}(d) \neq 0,
$$

or

$$
p \mid \operatorname{deg}(a) \text { and } p \mid \operatorname{deg}(d)
$$

For the former two, the computational effort decreases by a factor of $q$ if we disregard the computation of all the translates. Only for the last of these three types of pairs is the computational effort the same as in Algorithm 3.1. The exact proportion of pairs $(a, d)$ with $p \mid \operatorname{deg}(a)$ and $p \mid \operatorname{deg}(d)$ depends on the residue class of $\lfloor\operatorname{deg}(D) / 2\rfloor(\bmod p)$. Note that both $a$ and $d$ are non-zero since we only consider irreducible forms.

For brevity, we introduce the following terminology for cubic forms $f=(a, b, c, d)$. We call the form $f$ a

- type 1 form if $p \nmid \operatorname{deg}(a)$ and $\operatorname{sgn}_{2}(a)=0$, or $p \mid \operatorname{deg}(a)$, $p \nmid \operatorname{deg}(d)$ and $\operatorname{sgn}_{2}(d)=0$;

- type 2 form if $p \mid \operatorname{deg}(a)$ and $p \mid \operatorname{deg}(d)$;

- type 3 form otherwise, i.e., $p \nmid \operatorname{deg}(a)$ and $\operatorname{sgn}_{2}(a) \neq 0$, or $p \mid \operatorname{deg}(a), p \nmid \operatorname{deg}(d)$, and $\operatorname{sgn}_{2}(d) \neq 0$.

Note that, by Proposition 4.2, if $f=(a, b, c, d)$ is a type 1 form, then for all $\beta \in \mathbb{F}_{q}^{*}, f_{\beta}=(a(t+\beta), b(t+\beta), c(t+\beta), d(t+\beta))$ is a type 3 form. Moreover, all the $f_{\beta}$ for $\beta \in \mathbb{F}_{q}^{*}$ are pairwise distinct. Conversely, if $f=(a, b, c, d)$ is a type 3 form, then the forms $f_{\beta}$ for $\beta \in \mathbb{F}_{q}$ are pairwise distinct by Lemma $4.1(a(t)$ and $d(t)$ cannot both be polynomials in $t^{q}-t$ because $p$ does not divide both $\operatorname{deg}(a)$ and $\operatorname{deg}(d)$ in this case), and by Proposition 4.2, exactly one of the $f_{\beta}$ is a type 1 form and the others are type 3 forms.

The revised algorithm, given as Algorithm 4.4 below, only loops over forms of type 1 and 2 in steps 1-8 and incorporates the discriminant count arising from the type 3 forms via translates in step 11, whereas Algorithm 3.1 looped over forms of all three types. 
Each discriminant $D$ is endowed with two counters. One is the counter keeping track of the number of forms for each discriminant as in step 21 of Algorithm 3.1. The other one is a translate counter that counts how often $D$ is encountered as the discriminant of a type 1 form $f=(a, b, c, d)$. If $D(t)$ has translate counter $C_{D}$, then each $D(t+\beta)$ with $\beta \in \mathbb{F}_{q}^{*}$ occurs exactly $C_{D}$ times as the discriminant of the type 3 form $f_{\beta}=(a(t+\beta), b(t+\beta), c(t+\beta), d(t+\beta))$.

Algorithm 4.4. Improved 3-rank algorithm for computation of quadratic function fields where $-3 D$ is imaginary using Horner's rule for translates.

Input: A prime power $q$ not divisible by 2 or 3 , a primitive root $h$ of $\mathbb{F}_{q}$, the set $S=\left\{1, h, h^{2}, \ldots h^{(q-3) / 2}\right\}$, and a positive integer $B$.

Output: $A$ table where each entry is a square-free discriminant $D$ and a number of the form $\left(3^{r}-1\right) / 2$ with $-3 D$ imaginary, $\operatorname{sgn}(-3 D) \in$ $\{1, h\}$, and $\operatorname{deg}(D) \leq B$. The positive integer $r$ is the 3 -rank of the quadratic function field $\mathbb{F}_{q}(t, \sqrt{D})$.

1: for $\operatorname{deg}(a) \leq B / 4 \boldsymbol{A N D} \operatorname{sgn}(a) \in S \boldsymbol{A N D}\left(\operatorname{sgn}_{2}(a)=0\right.$ if $p \nmid \operatorname{deg}(a)$ OR $p \mid \operatorname{deg}(a)) \boldsymbol{d o}$

2: $\quad$ Execute Steps 2-14 of Algorithm 3.1

3: $\quad$ for $\operatorname{deg}(d)=i \boldsymbol{A N D}\left(\operatorname{sgn}_{2}(d)=0\right.$ if $\left.p \nmid \operatorname{deg}(d) \boldsymbol{O R} p \mid \operatorname{deg}(d)\right)$ do

4: $\quad$ Execute Steps 14-19 of Algorithm 3.1

5: $\quad$ if $\operatorname{deg}(D) \leq B \boldsymbol{A} \boldsymbol{N D}-3 D$ is imaginary (resp. unusual) $\boldsymbol{A N D}-3 D$ is fundamental $\boldsymbol{A N D} f$ is reduced then

6: $\quad$ Increment the 3-rank counter for discriminant $D$ by one in the hash table;

\%: $\quad$ if $p \nmid \operatorname{deg}(a)$ OR $p \nmid \operatorname{deg}(d)$ then

8: $\quad$ Increment the translate counter of $D(t)$ by 1

9: for all $D(t)$ in the hash table with positive translate counter do

10: $\quad$ Compute $D(t+\beta)$ for $\beta \in \mathbb{F}_{q}$ using Horner's Rule

11: Increase the counter for $D(t+\beta)$ by the value of the translate counter of $D(t)$

12: return hash table

Theorem 4.5. Algorithm 4.4 is correct.

Proof. We show that Algorithms 3.1 and 4.4 have exactly the same output. Steps 1-8 of Algorithm 4.4 loop exactly over all the type 1 
and 2 forms of degree up to $B$, and no type 3 form. Each type 1 form $f=(a, b, c, d)$ of discriminant $D(t)$ gives rise to $q-1$ type 3 forms $f_{\beta}=(a(t+\beta), b(t+\beta), c(t+\beta), d(t+\beta))$ of respective discriminants $D(t+\beta)$ for $\beta \in \mathbb{F}_{q}^{*}$ that all generate the same cubic field as $f$. Steps 9-11 generate all these discriminants, and, if $D(t)$ has translate counter $C_{D}$, i.e., is the discriminant of $C_{D}$ forms $f$ of type 1 , then each $D(t+\beta)$ is (in addition to the current value of the counter of $D(t+\beta)$ ) the discriminant of the $C_{D}$ forms $f_{\beta}$ of type 3 . So, every discriminant output by Algorithm 4.4 is also output by Algorithm 3.1, and with the same 3-rank count.

Conversely, let $D$ be a discriminant together with a count that is produced by step 21 of Algorithm 3.1. Let $D(t)$ be the discriminant of $C_{j}$ type $j$ forms for $j=1,2,3$. Since steps $1-8$ of Algorithm 4.4 only loop over all type 1 and 2 forms, and no type 3 forms, it produces $D$ with counter $C_{1}+C_{2}$. Now, each of the $C_{3}$ occurrences of $D(t)$ as the discriminant of a type 3 form $f_{i}=\left(a_{i}, b_{i}, c_{i}, d_{i}\right)$ corresponds to exactly one occurrence of some translate of $D(t)$ that is the discriminant of a type 1 form as follows. If $p \nmid \operatorname{deg}\left(a_{i}\right)$ and $\operatorname{sgn}_{2}\left(a_{i}\right) \neq 0$, then there exists a unique $\beta_{i} \in \mathbb{F}_{q}^{*}$ such that $\operatorname{sgn}_{2}\left(a_{i}\left(t-\beta_{i}\right)\right)=0$ by Proposition 4.2. Then

$$
f_{i}^{\prime}=\left(a_{i}\left(t-\beta_{i}\right), b_{i}\left(t-\beta_{i}\right), c_{i}\left(t-\beta_{i}\right), d_{i}\left(t-\beta_{i}\right)\right)
$$

is a type 1 form of discriminant $D_{i}^{\prime}(t)=D\left(t-\beta_{i}\right)$. If $p \mid \operatorname{deg}\left(a_{i}\right), p \nmid$ $\operatorname{deg}\left(d_{i}\right)$, and $\operatorname{sgn}_{2}\left(d_{i}\right) \neq 0$, then there again exists a unique $\beta_{i} \in \mathbb{F}_{q}^{*}$ such that $\operatorname{sgn}_{2}\left(d_{i}\left(t-\beta_{i}\right)\right)=0$. Then $f_{i}^{\prime}=\left(a_{i}\left(t-\beta_{i}\right), b_{i}\left(t-\beta_{i}\right), c_{i}(t-\right.$ $\left.\left.\beta_{i}\right), d_{i}\left(t-\beta_{i}\right)\right)$ is again a type 1 form of discriminant $D_{i}^{\prime}(t)=D\left(t-\beta_{i}\right)$. Note that these two possibilities are mutually exclusive. For either case, $D(t)=D_{i}^{\prime}\left(t+\beta_{i}\right)$ is encountered in steps 9-11 of Algorithm 4.4. Now each $D_{i}^{\prime}(t)$ occurred exactly $C_{D_{i}^{\prime}}$ times as the discriminant of a type 1 form, and these type 1 forms are exactly the forms $f_{i}^{\prime}$. So $D(t)=D_{i}^{\prime}\left(t+\beta_{i}\right)$ occurs exactly $C_{D_{i}^{\prime}}$ times as the discriminant of the corresponding type 3 form, and these forms are exactly the forms $f_{i}$.

5. Complexity of the improved algorithm. We now analyze the complexity of Algorithm 4.4. As before, let $p$ denote the characteristic of $\mathbb{F}_{q}$. As in [28], denote by $\mathcal{F}_{s}$ the set of binary cubic forms $f=$ $(a, b, c, d)$ over $\mathbb{F}_{q}[t]$ such that $\operatorname{deg}(D(f))=s, \operatorname{deg}(a) \leq s / 4, \operatorname{deg}(b) \leq$ $s / 4, \operatorname{deg}(a d) \leq s / 2, \operatorname{deg}(b c) \leq s / 2$, and $\operatorname{sgn}(a) \in S$. Using the fact that 
there are $(q-1) / 2$ choices for $\operatorname{sgn}(a), q-1$ choices for $\operatorname{sgn}(d)$, and $q$ choices each for the highest permissable coefficient of $b$ and $c$, we recall from [28, Lemma 7.1] that

$$
\# \mathcal{F}_{s}=\frac{q^{4-\delta_{s}}}{32} s^{2} q^{s}+O\left(q^{s}\right)
$$

as $s \rightarrow \infty$, where $\delta_{s}$ is the parity of $s$. Using this result, [28, Corollary 7.2] established a run time, using the bound $B$ on discriminant degrees, for the cubic function field tabulation algorithm ([28, Algorithm 2]), and hence also for Algorithm 3.1, of $O\left(B^{4} q^{B}\right)$ operations in $\mathbb{F}_{q}$ as $B \rightarrow \infty$. Disregarding constants arising from polynomial arithmetic, the dominant term of the $O$ constant when viewed as a polynomial in $q$ was $q^{3} / 16$ when $B$ is odd and $q^{4} / 32$ when $B$ is even. Our modifications herein improve this constant by a factor of $q$ most of the time; only when $B \equiv 0,1(\bmod 2 p)$ is the speed up smaller, but still significant. We begin with an auxiliary lemma.

Lemma 5.1. Let $m, n, r \in \mathbb{N}$ with $m \leq n$, and set

$$
N(m, n, r)=\sum_{i \leq m} \sum_{i+j \leq n} r^{i+j}
$$

Then

$$
N(m, n, r)=\frac{r}{r-1} m r^{n}+O\left(r^{n}\right) \quad \text { as } m \rightarrow \infty .
$$

Proof.

$$
N(m, n, r)=\sum_{i=0}^{m} r^{i} \sum_{j=0}^{n-i} r^{j}=\sum_{i=0}^{m} \frac{r^{n+1}-r^{i}}{r-1}=\frac{r}{r-1} m r^{n}+O\left(r^{n}\right),
$$

as claimed.

Corollary 5.2. For $s \geq 0$, let $P(s)$ denote the number of pairs of polynomials $(G, H)$ where $G, H \in \mathbb{F}_{q}[t], G$ and $H$ are monic, $\operatorname{deg}(G) \leq s / 4$ and $\operatorname{deg}(G H) \leq s / 2$. Then

$$
P(s)=\frac{q}{4(q-1)} s q^{\lfloor s / 2\rfloor}+O\left(q^{s / 2}\right)
$$

as $s \rightarrow \infty$. 
Proof. The number of monic polynomials in $\mathbb{F}_{q}[t]$ of degree at most $i$ is $q^{i}$. Hence,

$$
P(s)=\sum_{i \leq s / 4} \sum_{i+j \leq s / 2} q^{i+j}=N(s / 4,\lfloor s / 2\rfloor, q) .
$$

The result now follows from Lemma 5.1.

Corollary 5.3. For $s \geq 0$, let $Q(s)$ denote the number of pairs of polynomials $(G, H)$ where $G, H \in \mathbb{F}_{q}[t], G$ and $H$ are monic, $p|\operatorname{deg}(G), p| \operatorname{deg}(H), \operatorname{deg}(G) \leq s / 4$ and $\operatorname{deg}(G H) \leq s / 2$. Then

$$
Q(s)=\frac{q^{p}}{4 p\left(q^{p}-1\right)} s q^{\lfloor s / 2\rfloor-r_{s}}+O\left(q^{s / 2}\right)
$$

as $s \rightarrow \infty$, where $\lfloor s / 2\rfloor \equiv r_{s}(\bmod p)$ with $0 \leq r_{s} \leq p-1$.

Proof. We have

$$
\begin{aligned}
Q(s) & =\sum_{p i \leq s / 4} \sum_{p i+p j \leq s / 2} q^{p i+p j}=N\left(s / 4 p,\lfloor s / 2 p\rfloor, q^{p}\right) \\
& =\frac{q^{p}}{4 p\left(q^{p}-1\right)} s q^{p\lfloor s / 2 p\rfloor}+O\left(q^{s / 2}\right) .
\end{aligned}
$$

It remains to show that $p\lfloor s / 2 p\rfloor=\lfloor s / 2\rfloor-r_{s}$ to deduce the claim from Lemma 5.1. Let $\delta_{s}$ denote the parity of $s$, so $\lfloor s / 2\rfloor=\left(s-\delta_{s}\right) / 2$. Since

$$
\frac{s}{2 p}-\frac{s-\delta_{s}}{2 p}=\frac{\delta_{s}}{2 p}, \quad 0 \leq \frac{\delta_{s}}{2 p}<1,
$$

we see that $\lfloor s / 2 p\rfloor=\left\lfloor\left(s-\delta_{s}\right) / 2 p\right\rfloor$, and hence, by the definition of $r_{s}$,

$$
\begin{aligned}
\left\lfloor\frac{s}{2}\right\rfloor-r_{s} & =p\left\lfloor\frac{\lfloor s / 2\rfloor}{p}\right\rfloor=p\left\lfloor\frac{\left(s-\delta_{s}\right) / 2}{p}\right\rfloor \\
& =p\left\lfloor\frac{s-\delta_{s}}{2 p}\right\rfloor=p\left\lfloor\frac{s}{2 p}\right\rfloor,
\end{aligned}
$$

as desired.

Lemma 5.4. For any $s$, the number of type 1 forms in $\mathcal{F}_{s}$ is

$$
N_{1}(s)=\frac{(q-1)^{2} q}{2} P(s)(P(s)-Q(s)),
$$


where $P(s)$ and $Q(s)$ are defined as in Corollaries 5.2 and 5.3, respectively.

Proof. The number of pairs of polynomials $(G, H)$ defined in Corollary 5.2 with $p \nmid \operatorname{deg}(G)$ or $p \nmid \operatorname{deg}(H)$ is $P(s)-Q(s)$. For those pairs with $p \nmid \operatorname{deg}(G)$, a proportion of $1 / q$ has $\operatorname{sgn}_{2}(G)=0$, and for those with $p \mid \operatorname{deg}(G)$ and $p \nmid \operatorname{deg}(H)$, a proportion of $1 / q$ has $\operatorname{sgn}_{2}(H)=0$. It follows that the number of pairs $(a, d)$ for which $f=(a, b, c, d)$ is a type 1 form in $\mathcal{F}_{s}$ is

$$
\frac{(q-1)^{2}}{2 q}(P(s)-Q(s)),
$$

and the number of $(b, c)$ pairs for such a form is $q^{2} P(s)$. Multiplying these two counts yields the desired result.

Lemma 5.5. For any $s$, the number of type 2 forms in $\mathcal{F}_{s}$ is

$$
N_{2}(s)=\frac{(q-1)^{2} q^{2}}{2} P(s) Q(s),
$$

where $P(s)$ and $Q(s)$ are defined as in Corollaries 5.2 and 5.3, respectively.

Proof. The number of $(a, d)$ pairs for which $f=(a, b, c, d)$ is a type 2 form in $\mathcal{F}_{s}$ is $(q-1)^{2} Q(s) / 2$, and the number of $(b, c)$ pairs for such a form is again $q^{2} P(s)$.

Corollary 5.6. The combined number of type 1 and 2 forms in $\mathcal{F}_{s}$ is

$$
\begin{aligned}
N_{1}(s)+N_{2}(s)= & \frac{q^{3-\delta_{s}}}{32}\left(\frac{(q-1)^{2}}{p q^{1+r_{s}}} \frac{q^{p}}{q^{p}-1}+1\right) s^{2} q^{s} \\
& +O\left(s q^{s}\right) .
\end{aligned}
$$

Proof. By Lemmas 5.4 and 5.5, the combined number of type 1 and 2 forms $\mathcal{F}_{s}$ is

$$
\begin{aligned}
N_{1}(s)+N_{2}(s) & =\frac{(q-1)^{2} q}{2} P(s)(P(s)-Q(s)+q Q(s)) \\
& =\frac{(q-1)^{2} q}{2}\left(P(s)^{2}+(q-1) P(s) Q(s)\right) .
\end{aligned}
$$


We evaluate each term separately. As before, let $\delta_{s}$ be the parity of $s$, so that $2\lfloor s / 2\rfloor=s-\delta_{s}$. Then

$$
\begin{aligned}
\frac{(q-1)^{2} q}{2} P(s)^{2} & =\frac{(q-1)^{2} q}{2} \frac{q^{2}}{16(q-1)^{2}} s^{2} q^{s-\delta_{s}}+O\left(s q^{s}\right) \\
& =\frac{q^{3-\delta_{s}}}{32} s^{2} q^{s}+O\left(s q^{s}\right) .
\end{aligned}
$$

Setting $\left(s-\delta_{s}\right) / 2 \equiv r_{s}(\bmod p)$ with $0 \leq r_{s} \leq p-1$ as before, we also obtain

$$
\begin{aligned}
\frac{(q-1)^{3} q}{2} P(s) Q(s)= & \frac{(q-1)^{3} q}{2} \frac{q}{16(q-1)} \frac{q^{p}}{4 p\left(q^{p}-1\right)} s^{2} q^{s-\delta_{s}-r_{s}} \\
& +O\left(s q^{s}\right) \\
= & \frac{(q-1)^{2} q^{2-\delta_{s}-r_{s}}}{32 p} \frac{q^{p}}{q^{p}-1} s^{2} q^{s}+O\left(s q^{s}\right) .
\end{aligned}
$$

Altogether,

$$
N_{1}(s)+N_{2}(s)=\frac{q^{3-\delta_{s}}}{32}\left(1+\frac{(q-1)^{2}}{p q^{1+r_{s}}} \frac{q^{p}}{q^{p}-1}\right) s^{2} q^{s}+O\left(s q^{s}\right) .
$$

Note that the factor $q^{p} /\left(q^{p}-1\right)$ is extremely close to 1 , even for small values of $p$ and $q$. For the smallest permissible parameters, when $p=q=5$, this quantity is approximately 1.0003 ; for $q=p=7$, it is roughly 1.000001 .

Theorem 5.7. Assuming standard polynomial arithmetic in $\mathbb{F}_{q}[t]$, Algorithm 4.4 requires $O\left(B^{4} q^{B}\right)=O\left(q^{B+\epsilon}\right)$ operations in $\mathbb{F}_{q}$ as $B \rightarrow$ $\infty$. Here, the $O$-constant is a rational function of $q$ whose dominant term is of order $q^{4-\delta_{B}} / p$ if $B \equiv 0,1(\bmod 2 p)$ and of order $q^{3-\delta_{B}}$ otherwise, where $\delta_{B}$ is the parity of $B$.

Proof. The analysis of steps 1-8 of the algorithm proceeds analogously to [28, Corollary 7.3]. These steps run over all the type 1 and type 2 forms in $\mathcal{F}_{s}$ for $3 \leq s \leq B$. For each such form, the entire collection of polynomial computations requires at most $K s^{2}$ field operations for some constant $K$ that is independent of $B$ and $q$. This holds because all polynomials under consideration have degree bounded by 
$s$. So the asymptotic run time of steps $1-8$ is

$$
\begin{aligned}
T_{1}(B) & =\sum_{s=3}^{B}\left(\left(N_{1}(s)+N_{2}(s)\right) \cdot K s^{2}\right) \\
& =K \sum_{s=3}^{B}\left(C_{s} s^{4} q^{s}+O\left(s^{3} q^{s}\right)\right) \\
& =K B^{4} \sum_{s=3}^{B} C_{s} q^{s}+O\left(B^{3} q^{B}\right),
\end{aligned}
$$

where $N_{1}(s)$ and $N_{2}(s)$ are given by Lemmas 5.4 and 5.5, respectively, and

$$
C_{s}=\frac{q^{3-\delta_{s}}}{32}\left(\frac{(q-1)^{2}}{p q^{1+r_{s}}} \frac{q^{p}}{q^{p}-1}+1\right)
$$

by Corollary 5.6. Since $\delta_{s} \geq 0, r_{s} \geq 0$ and $q-1<q$, we see that $C_{s}<C(q)$ where

$$
C(q)=\frac{q^{4}}{32 p} \frac{q^{p}}{q^{p}-1} \text {. }
$$

It follows that

$$
\begin{aligned}
\sum_{s=3}^{B} C_{s} q^{s} & <C(q) \sum_{s=0}^{B-2} q^{s}+C_{B-1} q^{B-1}+C_{B} q^{B} \\
& <\left(\frac{C(q)}{q(q-1)}+\frac{C_{B-1}}{q}+C_{B}\right) q^{B} .
\end{aligned}
$$

The dominant term in $C(q) /(q(q-1))$ is $q^{2} / 32 p$.

If $B$ is even, then $r_{B-1} \neq 0$, so

$$
\frac{C_{B-1}}{q}+C_{B}<\frac{q}{32}+\frac{q^{3}}{32}\left(\frac{q^{1-r_{B}}}{p} \frac{q^{p}}{q^{p}-1}+1\right) .
$$

The dominant term in this constant is $q^{3}\left(q^{1-r_{B}} / p+1\right) / 32$. This is of order $q^{4} / p$ if $r_{B}=0$, i.e., $B \equiv 0(\bmod 2 p)$, and $q^{3}$ otherwise.

If $B$ is odd, then $r_{B} \neq 0$, and hence $r_{B-1}=r_{B}-1$. Hence,

$$
\frac{C_{B-1}}{q}+C_{B}<\frac{q^{2}}{32}\left(\frac{q^{2-r_{B}}}{p} \frac{q^{p}}{q^{p}-1}+1\right)+\frac{q^{2}}{32}
$$




$$
=\frac{q^{2}}{32}\left(\frac{q^{2-r_{B}}}{p} \frac{q^{p}}{q^{p}-1}+2\right) .
$$

The dominant term in this constant is $q^{2}\left(q^{2-r_{B}} / p+2\right) / 32$. This is of order $q^{3} / p$ if $r_{B}=1$, i.e., $B \equiv 1(\bmod 2 p)$, and $q^{2}$ otherwise.

Next, we analyze steps 9-11 of Algorithm 4.4 and show that their run time is negligible compared to that of steps 1-8. For any given degree $s$ the number of discriminants of degree $s$ that steps 9-11 loop over is certainly bounded above by the number of fundamental imaginary discriminants of degree $s$, which is $2(q-1) q^{s-1}$, by our remarks at the beginning of Section 8 . This is a very crude upper bound on the number of $D$ in the table, but it suffices for our purposes.

The run time of steps $9-11$ is dominated by step 10. Each translate $D(t+\beta)$ with $\beta \in \mathbb{F}_{q}^{*}$ can be computed by applying Horner's rule as follows: if $D(t)=a_{s} t^{s}+\cdots+a_{0}$, then initialize $D_{0}=a_{s}$ and compute $D_{i}=t D_{i-1}+\beta D_{i-1}+a_{s-i}$ for $1 \leq i \leq s$. Then $D_{s}=D(t+\beta)$. For each translate $D(t+\beta)$, this requires $s$ shifts (whose cost is negligible) and $L s^{2}$ operations in $\mathbb{F}_{q}$ for some constant $L$ that is independent of $s$ and $q$. Since there are $q$ choices for $\beta$, the total number of field operations required by steps $9-11$ is no more than

$$
T_{2}(B)=\sum_{s=3}^{B} 2(q-1) q^{s-1} q L s^{2}=O\left(B^{2} q^{B}\right),
$$

which is asymptotically negligible compared to $T_{1}(B)$.

If $q=p$, then it is most efficient to evaluate $D(t+1)$ from $D(t)$ via $D_{0}=a_{s}$ and $D_{i}=t D_{i-1}+D_{i-1}+a_{s-i}$ for $1 \leq i \leq s$. Applying this technique repeatedly $p-1$ times generates all the polynomials $D(t+j)$ for $0 \leq j \leq p-1$ using only field additions, no multiplications.

In Tables 1 and 2, we compare the $O$-constants for the run time of Algorithm 3.1 as established in [28, Corollary 7.3] with those derived in the proof of Theorem 5.7. We disregard the constants arising from polynomial arithmetic (denoted above by $K$ and $L$ ).

6. Conjectures and results on class group distributions. In this section, we recall the Friedman-Washington heuristics and other results on the distribution of class groups of function fields, in order to compare the data from our algorithm to these conjectures and provide 
TABLE 1. Comparison of the O-constants in the run times of Algorithms 3.1 and $4.4, q \neq p$.

\begin{tabular}{|c|c|c|c|c|}
\hline Parity of $B$ & $B(\bmod 2 p)$ & Alg. 3.1 & Alg. 4.4 & Speed-up factor \\
\hline \hline$B$ odd & $B \not \equiv 1$ & $q^{3} / 16$ & $q^{2} / 16$ & $q$ \\
$B$ odd & $B \equiv 1$ & $q^{3} / 16$ & $q^{3} / 32 p$ & $2 p$ \\
\hline$B$ even & $B \not \equiv 0$ & $q^{4} / 32$ & $q^{3} / 32$ & $q$ \\
$B$ even & $B \equiv 0$ & $q^{4} / 32$ & $q^{4} / 32 p$ & $p$ \\
\hline
\end{tabular}

TABlE 2. Comparison of the O-constants in the run times of Algorithms 3.1 and $4.4, q=p$.

\begin{tabular}{|c|c|c|c|c|}
\hline Parity of $B$ & $B(\bmod 2 p)$ & Alg. 3.1 & Alg. 4.4 & Speed-up factor \\
\hline \hline$B$ odd & $B \neq \equiv 1$ & $q^{3} / 16$ & $q^{2} / 16$ & $q$ \\
$B$ odd & $B \equiv 1$ & $q^{3} / 16$ & $3 q^{2} / 32$ & $2 q / 3$ \\
\hline$B$ even & $B \neq \equiv 0$ & $q^{4} / 32$ & $q^{3} / 32$ & $q$ \\
$B$ even & $B \equiv 0$ & $q^{4} / 32$ & $q^{3} / 16$ & $q / 2$ \\
\hline
\end{tabular}

some numerical validity supporting them in Section 7 . Before we give a more detailed description of each $\ell$-rank heuristic/result, we provide a brief overview of their respective underlying assumptions (in addition to requiring that $\ell$ be odd in all cases):

- Friedman-Washington [14]. $q \not \equiv 0(\bmod \ell)$, imaginary quadratic extensions;

- Ellenberg et al. [12]. $q \not \equiv 0,1(\bmod \ell)$, imaginary quadratic extensions;

- Achter [1]. $q \equiv 1(\bmod \ell)$, imaginary or unusual quadratic extensions;

- Garton $[\mathbf{1 6}, \mathbf{1 7}] . q \equiv 1(\bmod \ell), q \not \equiv 1\left(\bmod \ell^{2}\right)$, imaginary quadratic extensions.

All the aforementioned results apply to Jacobians (i.e., degree zero divisor class groups) of hyperelliptic curves. It is well known that the Jacobian and the ideal class group of an imaginary or unusual hyperelliptic function field are very closely linked; they are essentially isomorphic (possibly up to a factor of $\mathbb{Z} / 2 \mathbb{Z}$ ), so their respective $\ell$-ranks are equal when $\ell$ is odd. Therefore, we can use the ideal class group when comparing our data to the heuristics without loss of generality. 
We now give an in-depth description of each result. The FriedmanWashington heuristics [14] are entirely analogous to the Cohen-Lenstra heuristics. Loosely speaking, Friedman and Washington predict that, given a fixed finite field $\mathbb{F}_{q}$ and an abelian $\ell$-group $A$, where $\ell$ is an odd prime that does not divide $q, A$ occurs as the $\ell$-Sylow part of the divisor class group of a quadratic function field over $\mathbb{F}_{q}$ with frequency inversely proportional to $|\operatorname{Aut}(A)|$. The precise statement is given below. For brevity, define, for any $n, r \in \mathbb{N}$ the quantities

$$
\eta_{r}(n)=\prod_{j=1}^{r}\left(1-n^{-j}\right) \quad \text { and } \quad \eta_{\infty}(n)=\prod_{j \geq 1}\left(1-n^{-j}\right) .
$$

Conjecture 6.1 (Friedman and Washington [14]). Let $\ell$ be an odd prime not dividing $q$. Then a finite abelian group $A$ of $\ell$-power order appears as the $\ell$-Sylow part $C l_{\ell}$ of the class group of an imaginary quadratic extension $K$ of $\mathbb{F}_{q}(t)$ of genus $g_{K}$ with a frequency inversely proportional to the number of automorphisms of $A$. That is,

$$
\lim _{g \rightarrow \infty}\left(\sum_{\substack{K \\ g_{K} \leq g \\ C l_{\ell} \cong A}} 1 / \sum_{\substack{K \\ g_{K} \leq g}} 1\right)=\frac{\eta_{\infty}(\ell)}{|\operatorname{Aut}(A)|} .
$$

A newer, related result, due to Ellenberg, Venkatesh and Westerland $[\mathbf{1 2}, \mathbf{3 2}]$, states that the upper and lower densities of imaginary quadratic extensions of $\mathbb{F}_{q}(t)$ for which the $\ell$-part of the class group is isomorphic to any given finite abelian $\ell$-group converges to the righthand side of equation (6.1), as $q \rightarrow \infty$ with $q \not \equiv 0,1(\bmod \ell)$.

Theorem 6.2 (Ellenberg, Venkatesh and Westerland [12]). Let $\ell$ be an odd prime and $A$ a finite abelian $\ell$-group. Write $\delta^{+}(q)$ (respectively, $\delta^{-}(q)$ ) for the upper density (respectively, lower density) of imaginary quadratic extensions of $\mathbb{F}_{q}(t)$ for which the $\ell$-part of the class group is isomorphic to $A$. Then $\delta^{+}(q)$ and $\delta^{-}(q)$ converge, as $q \rightarrow \infty$ with $q \not \equiv 0,1(\bmod \ell)$, to $\eta_{\infty}(\ell) /|\operatorname{Aut}(A)|$.

The result of Theorem 6.2 requires the additional assumption that $q \rightarrow \infty$, but the predicted distribution is what Friedman and Washington assert for fixed $q \not \equiv 0,1(\bmod \ell)$. We note that, for fixed values 
of $q$, the Friedman-Washington heuristic is still a conjecture. Based on Conjecture 6.1 and Theorem 6.2, the probability that the $\ell$-rank of an ideal class group of an imaginary quadratic function field is equal to $r$, as given in Cohen and Lenstra [9] for number fields and in Lee [22] for function fields, is given by

$$
\frac{\eta_{\infty}(\ell)}{\eta_{r}(\ell)^{2} \ell^{r^{2}}}
$$

For $\ell=3$ and the values of $r=0,1,2,3,4$, we obtain the approximate probabilities $0.56128,0.42009,0.019692,0.00008739$ and $4.0964 \times 10^{-8}$, respectively.

Achter $[\mathbf{1}, \mathbf{2}]$ proved a version of the Friedman-Washington heuristic where $q \rightarrow \infty$ inside $\lim _{g \rightarrow \infty}$ with $q \equiv 1(\bmod \ell)$, and the quadratic extension is assumed to have one infinite place. His result is an explicit formula in terms of $\ell$ for the number

$$
\alpha(g, r)=\frac{\left|\left\{x \in \mathrm{Sp}_{2 g}(\mathbb{F}): \operatorname{ker}(x-i d) \simeq \mathbb{F}^{r}\right\}\right|}{\left|\mathrm{Sp}_{2 g}(\mathbb{F})\right|},
$$

where $\mathrm{Sp}_{2 g}(\mathbb{F})$ denotes the group of $2 g$ by $2 g$ symplectic matrices over a field $\mathbb{F}$ or order $\ell$. The function $\alpha(g, r)$ expresses similar probabilities as in the original Friedman-Washington heuristic in the case $q \equiv 1$ $(\bmod \ell)$. Achter's result $[\mathbf{1}]$ proves a weaker version of Friedman and Washington's original conjecture since it requires $q \rightarrow \infty$ in addition to $g \rightarrow \infty$.

Empirical evidence (see Malle $[\mathbf{2 4}, \mathbf{2 5}]$ ) strongly suggests that the presence of $\ell$ th roots of unity in the base field skews the distribution of $\ell$-rank values of quadratic number fields. In order to account for this discrepancy for number fields, Malle [25] proposed alternative conjectural formulas to cover the case where primitive $\ell$ th roots of unity lie in the base field, provided primitive $\ell^{2}$ th roots of unity that are themselves not also $\ell$ th roots of unity do not lie in the base field.

A different probability distribution, one consistent with Malle's conjectures $[\mathbf{2 4}, \mathbf{2 5}]$ and Achter's results $[\mathbf{1}, \mathbf{2}]$, is obtained for $q \equiv 1$ $(\bmod \ell)$ due to the presence of $\ell$ th roots of unity. The analogous weak Friedman-Washington result in this case is due to Garton $[\mathbf{1 6}, \mathbf{1 7}]$. Garton's result, again with $q \rightarrow \infty$ as well as $g \rightarrow \infty$, predicts that the upper and lower densities of imaginary quadratic extensions of $\mathbb{F}_{q}(t)$ for which the $\ell$-part of the class group is isomorphic to any given 
finite abelian $\ell$-group converges to Malle's formula for number fields, as $q \rightarrow \infty$ with $q \equiv 1(\bmod \ell)$. This is summarized in the following theorem.

Theorem 6.3 (Garton $[\mathbf{1 6}, \mathbf{1 7}]$ ). Let $\ell$ be an odd prime and $A$ a finite abelian $\ell$-group. Write $\delta^{+}(q)$ (respectively, $\left.\delta^{-}(q)\right)$ for the upper density (respectively, lower density) of imaginary quadratic extensions of $\mathbb{F}_{q}(t)$ for which the $\ell$-part of the class group is isomorphic to $A$. Then $\delta^{+}(q)$ and $\delta^{-}(q)$ converge, as $q \rightarrow \infty$ with $q \equiv 1(\bmod \ell)$ and $q \neq \equiv 1\left(\bmod \ell^{2}\right)$, to

$$
\frac{\eta_{\infty}(\ell) \eta_{r}(\ell) \ell^{r(r-1) / 2}}{\eta_{\infty}\left(\ell^{2}\right)|A u t(A)|}
$$

From the distribution given in Theorem 6.3 , the probability that a quadratic function field has $\ell$-rank equal to $r$ in the case $q \equiv 1(\bmod \ell)$ and $q \not \equiv 1\left(\bmod \ell^{2}\right)$, is given by

$$
\frac{\eta_{\infty}(\ell)}{\eta_{\infty}\left(\ell^{2}\right) \eta_{r}(\ell) \ell^{r(r+1) / 2}} .
$$

For $\ell=3$ and the values of $r=0,1,2,3,4$, we obtain the approximate probabilities $0.64032,0.31950,0.03994,1.5361 \times 10^{-3}$ and $1.9201 \times 10^{-5}$, respectively.

We note that Achter's function $\alpha$ converges to a formula of Malle for number fields as $g \rightarrow \infty$, giving additional evidence for a stronger version of Garton's result, where the dependence on $q \rightarrow \infty$ is removed. This result is Malle [25, Proposition 3.1]. In Section 7, we will use the numerical data we generated to see how well the data matches the Friedman and Washington and Ellenberg, Venkatesh and Westerland results for $q \equiv-1(\bmod \ell)$, and the Garton distribution result for $q \equiv 1$ $(\bmod \ell)$ and $q \neq \equiv 1\left(\bmod \ell^{2}\right)$ when $q$ is fixed.

7. Numerical results. Tables 3 and 4 present the results of our computations for the 3-rank counts of quadratic function fields for $q=5,7,11,13$ using Algorithm 4.4 for imaginary discriminants and Algorithm 3.1 for unusual discriminants $-3 D$ with $q=5,7$ (fewer fields in the unusual case due to the longer running times). For each of these $q$-values, $h=2$ was chosen as a primitive root of $\mathbb{F}_{q}$ with the exception of $\mathbb{F}_{7}$, where $h=3$ was chosen. This completely determines the set 
$S$ specified in Section 2. These sets were $\{1,2\},\{1,2,3\},\{1,2,4,5,8\}$ and $\{1,2,3,4,6,8\}$ for $\mathbb{F}_{5}, \mathbb{F}_{7}, \mathbb{F}_{11}$ and $\mathbb{F}_{13}$, respectively.

We implemented our counting algorithms (both Algorithms 3.1 and 4.4) using the $\mathrm{C}++$ programming language coupled with the number theory library NTL [30]. The lists of quadratic fields and their (positive) 3-ranks were computed on a 32 core $2.0 \mathrm{GHz}$ Intel Xeon X7550 machine running Unix with $256 \mathrm{~GB}$ of RAM. Each table entry consists of the base field size $q$, the degree bound on the discriminant $D$ and the corresponding genus $g$, the total number of square-free discriminants of that degree, the 3 -rank of $\mathbb{F}_{q}(t, \sqrt{D})$, the total number of fields with given $\operatorname{deg}(D)$ value found with that 3 -rank, and the total elapsed time to find all quadratic function fields with the given degree and 3-rank at least 1 .

Table 3: 3-ranks of quadratic function fields over $\mathbb{F}_{q}$ with $-3 D$ imaginary

\begin{tabular}{|c|c|c|c|c|c|}
\hline$q$ & $\operatorname{deg}(D), g$ & $\#$ of $D$ & 3-rank & Total & Time \\
\hline \multirow[t]{14}{*}{5} & 3,1 & 200 & 1 & 80 & $0.00 \mathrm{~s}$ \\
\hline & \multirow[t]{2}{*}{5,2} & \multirow[t]{2}{*}{5000} & 1 & 1600 & \multirow[t]{2}{*}{$0.18 \mathrm{~s}$} \\
\hline & & & 2 & 10 & \\
\hline & \multirow[t]{2}{*}{7,3} & \multirow[t]{2}{*}{125000} & 1 & 46840 & \multirow[t]{2}{*}{$5.45 \mathrm{~s}$} \\
\hline & & & 2 & 1180 & \\
\hline & \multirow[t]{3}{*}{9,4} & \multirow[t]{3}{*}{3125000} & 1 & 1297120 & \multirow[t]{3}{*}{$3 \mathrm{~m}, 22 \mathrm{~s}$} \\
\hline & & & 2 & 51300 & \\
\hline & & & 3 & 40 & \\
\hline & \multirow[t]{3}{*}{11,5} & \multirow[t]{3}{*}{78125000} & 1 & 31730080 & \multirow{3}{*}{$\begin{array}{c}2 \mathrm{~h}, 15 \mathrm{~m}, \\
26 \mathrm{~s}\end{array}$} \\
\hline & & & 2 & 1167200 & \\
\hline & & & 3 & 1880 & \\
\hline & \multirow[t]{3}{*}{13,6} & \multirow[t]{3}{*}{1953125000} & 1 & 806759000 & \multirow{3}{*}{$\begin{array}{c}3 \mathrm{~d}, 23 \mathrm{~h}, \\
40 \mathrm{~m}\end{array}$} \\
\hline & & & 2 & 33601470 & \\
\hline & & & 3 & 88680 & \\
\hline \multirow[t]{6}{*}{7} & \multirow[t]{2}{*}{3,1} & \multirow[t]{2}{*}{588} & 1 & 196 & \multirow[t]{2}{*}{$0.04 \mathrm{~s}$} \\
\hline & & & 2 & 14 & \\
\hline & \multirow[t]{2}{*}{5,2} & \multirow[t]{2}{*}{28812} & 1 & 8400 & \multirow[t]{2}{*}{$2.48 \mathrm{~s}$} \\
\hline & & & 2 & 588 & \\
\hline & \multirow[t]{2}{*}{7,3} & \multirow[t]{2}{*}{1411788} & 1 & 432348 & \multirow[t]{2}{*}{$2 \mathrm{~m}, 20 \mathrm{~s}$} \\
\hline & & & 2 & 42924 & \\
\hline \multicolumn{6}{|c|}{ Continued on next page } \\
\hline
\end{tabular}


Table 3 (continued from previous page).

\begin{tabular}{|c|c|c|c|c|c|}
\hline$q$ & $\operatorname{deg}(D), g$ & $\#$ of $D$ & 3-rank & Total & Time \\
\hline & & & 3 & 840 & \\
\hline & \multirow[t]{4}{*}{9,4} & \multirow[t]{4}{*}{69177612} & 1 & 21996478 & \multirow{4}{*}{$\begin{array}{c}2 \mathrm{~h}, 44 \mathrm{~m} \\
24 \mathrm{~s}\end{array}$} \\
\hline & & & 2 & 2675302 & \\
\hline & & & 3 & 90874 & \\
\hline & & & 4 & 588 & \\
\hline & \multirow[t]{4}{*}{11,5} & \multirow[t]{4}{*}{3389702988} & 1 & 1072738086 & \multirow{4}{*}{$\begin{array}{l}8 \mathrm{~d}, 14 \mathrm{~h}, \\
48 \mathrm{~m}, 9 \mathrm{~s}\end{array}$} \\
\hline & & & 2 & 126751170 & \\
\hline & & & 3 & 4078662 & \\
\hline & & & 4 & 27174 & \\
\hline \multirow[t]{9}{*}{11} & 3,1 & 2420 & 1 & 1100 & $0.51 \mathrm{~s}$ \\
\hline & \multirow[t]{2}{*}{5,2} & \multirow[t]{2}{*}{292820} & 1 & 110000 & \multirow[t]{2}{*}{$1 \mathrm{~m}, 12 \mathrm{~s}$} \\
\hline & & & 2 & 2970 & \\
\hline & \multirow[t]{3}{*}{7,3} & \multirow{3}{*}{35431220} & 1 & 14186480 & \multirow{3}{*}{$\begin{array}{c}2 \mathrm{~h}, 40 \mathrm{~m} \\
4 \mathrm{~s}\end{array}$} \\
\hline & & & 2 & 506220 & \\
\hline & & & 3 & 660 & \\
\hline & \multirow[t]{3}{*}{9,4} & \multirow[t]{3}{*}{4287177620} & 1 & 1796730320 & \multirow[t]{3}{*}{$24 \mathrm{~d}, 23 \mathrm{~h}$} \\
\hline & & & 2 & 81402640 & \\
\hline & & & 3 & 288200 & \\
\hline \multirow[t]{9}{*}{13} & \multirow[t]{2}{*}{3,1} & \multirow[t]{2}{*}{4056} & 1 & 1352 & \multirow[t]{2}{*}{$1.35 \mathrm{~s}$} \\
\hline & & & 2 & 130 & \\
\hline & \multirow[t]{3}{*}{5,2} & \multirow[t]{3}{*}{685464} & 1 & 209352 & \multirow[t]{3}{*}{$4 \mathrm{~m}, 25 \mathrm{~s}$} \\
\hline & & & 2 & 20046 & \\
\hline & & & 3 & 312 & \\
\hline & \multirow[t]{4}{*}{7,3} & \multirow[t]{4}{*}{115843416} & 1 & 36281076 & \multirow{4}{*}{$\begin{array}{c}14 \mathrm{~h}, 38 \mathrm{~m}, \\
34 \mathrm{sec}\end{array}$} \\
\hline & & & 2 & 4009330 & \\
\hline & & & 3 & 108108 & \\
\hline & & & 4 & 494 & \\
\hline
\end{tabular}

Table 4: 3-ranks of quadratic function fields over $\mathbb{F}_{q}$ with $-3 D$ unusual

\begin{tabular}{|c|c|r|r|r|c|}
\hline$q$ & $\operatorname{deg}(D), g$ & \# of $D$ & 3 -rank & Total & Time \\
\hline 5 & 4,1 & 500 & 1 & 200 & $0.54 \mathrm{~s}$ \\
\cline { 2 - 5 } & 6,2 & 12500 & 1 & 4780 & $12.17 \mathrm{~s}$ \\
\cline { 3 - 4 } & & 2 & 100 & \\
\hline \multicolumn{5}{|c}{ Continued on next page } \\
\hline
\end{tabular}


Table 4 (continued from previous page).

\begin{tabular}{|c|c|c|c|c|c|}
\hline$q$ & $\operatorname{deg}(D), g$ & $\#$ of $D$ & 3-rank & Total & Time \\
\hline & \multirow[t]{2}{*}{8,3} & \multirow[t]{2}{*}{312500} & 1 & 115460 & \multirow[t]{2}{*}{$7 \mathrm{~m}, 49 \mathrm{~s}$} \\
\hline & & & 2 & 2205 & \\
\hline & \multirow[t]{3}{*}{10,4} & \multirow[t]{3}{*}{7812500} & 1 & 3240340 & \multirow{3}{*}{$\begin{array}{c}3 \mathrm{~h}, 9 \mathrm{~m} \\
18 \mathrm{~s}\end{array}$} \\
\hline & & & 2 & 128160 & \\
\hline & & & 3 & 100 & \\
\hline \multirow[t]{8}{*}{7} & \multirow[t]{2}{*}{4,1} & \multirow[t]{2}{*}{2058} & 1 & 672 & \multirow[t]{2}{*}{$7.08 \mathrm{~s}$} \\
\hline & & & 2 & 42 & \\
\hline & \multirow[t]{3}{*}{6,2} & \multirow[t]{3}{*}{100842} & 1 & 30989 & \multirow[t]{3}{*}{$5 \mathrm{~m}, 43 \mathrm{~s}$} \\
\hline & & & 2 & 3115 & \\
\hline & & & 3 & 63 & \\
\hline & \multirow[t]{3}{*}{8,3} & \multirow[t]{3}{*}{4941258} & 1 & 1510026 & \multirow{3}{*}{$\begin{array}{c}6 \mathrm{~h}, 36 \mathrm{~m} \\
21 \mathrm{~s}\end{array}$} \\
\hline & & & 2 & 142632 & \\
\hline & & & 3 & 2310 & \\
\hline
\end{tabular}

Timings for Algorithm 4.4 are compared to those of Algorithm 3.1 in Table 5 for $-3 D$ imaginary with $q=5,7,11,13$. As seen in the last column, the modified algorithm is a significant improvement over the basic algorithm, which becomes more pronounced as $q$ increases. This is expected as the complexity analysis for Algorithm 4.4 predicts an improvement in timings by roughly a factor of $q$, with an improvement of $2 q / 3$ for degree 11 fields over $\mathbb{F}_{5}$. The actual speed up is below the factor predicted by Table 5, but this is likely due to the fact that our degree bounds are too small for the asymptotics to take effect, so the error terms have a significant effect on the run times.

Table 5: Timings of Algorithm 3.1 versus Algorithm 4.4

\begin{tabular}{|c|c|c|c|c|}
\hline$q$ & Deg. bd. & Alg. 3.1 times & Alg. 4.4 times & $3.1 / 4.4$ \\
\hline 5 & 3 & $0.01 \mathrm{~s}$ & $0.00 \mathrm{~s}$ & - \\
\cline { 2 - 5 } & 5 & $0.59 \mathrm{~s}$ & $0.18 \mathrm{~s}$ & 3.28 \\
\cline { 2 - 5 } & 7 & $17.33 \mathrm{~s}$ & $5.45 \mathrm{~s}$ & 2.99 \\
\cline { 2 - 5 } & 9 & $11 \mathrm{~m}, 59 \mathrm{~s}$ & $3 \mathrm{~m}, 22 \mathrm{~s}$ & 3.57 \\
\cline { 2 - 5 } & 11 & $5 \mathrm{~h}, 5 \mathrm{~m}, 19 \mathrm{~s}$ & $2 \mathrm{~h}, 15 \mathrm{~m}, 26 \mathrm{~s}$ & 2.25 \\
\cline { 2 - 5 } & 13 & - & $\approx 4 \mathrm{~d}$ & - \\
\hline \multicolumn{4}{|c|}{ Continued on next page } \\
\hline
\end{tabular}


Table 5 (continued from previous page).

\begin{tabular}{|c|c|c|c|c|}
\hline$q$ & Degree bd. & Alg. 3.1 times & Alg. 4.4 times & $3.1 / 4.4$ \\
\hline 7 & 3 & $0.09 \mathrm{~s}$ & $0.04 \mathrm{~s}$ & 2.25 \\
\cline { 2 - 5 } & 5 & $10.75 \mathrm{~s}$ & $2.48 \mathrm{~s}$ & 4.33 \\
\cline { 2 - 5 } & 7 & $10 \mathrm{~m}, 2 \mathrm{~s}$ & $2 \mathrm{~m}, 20 \mathrm{~s}$ & 4.30 \\
\cline { 2 - 5 } & 9 & $13 \mathrm{~h}, 47 \mathrm{~m}, 6 \mathrm{~s}$ & $2 \mathrm{~h}, 44 \mathrm{~m}, 24 \mathrm{~s}$ & 5.03 \\
\cline { 2 - 5 } & 11 & - & $\approx 9 \mathrm{~d}$ & - \\
\hline 11 & 3 & $1.34 \mathrm{~s}$ & $0.51 \mathrm{~s}$ & 2.63 \\
\cline { 2 - 5 } & 5 & $6 \mathrm{~m}, 43 \mathrm{~s}$ & $1 \mathrm{~m}, 12 \mathrm{~s}$ & 5.58 \\
\cline { 2 - 5 } & 7 & $17 \mathrm{~h}, 2 \mathrm{~m}, 52 \mathrm{~s}$, & $2 \mathrm{~h}, 40 \mathrm{~m}, 4 \mathrm{~s}$ & 6.39 \\
\cline { 2 - 5 } & 9 & - & $\approx 25 \mathrm{~d}$ & - \\
\hline 13 & 3 & $4.26 \mathrm{~s}$ & $1.35 \mathrm{~s}$ & 3.16 \\
\cline { 2 - 5 } & 5 & $25 \mathrm{~m}, 44 \mathrm{~s}$ & $4 \mathrm{~m}, 25 \mathrm{~s}$ & 5.81 \\
\cline { 2 - 5 } & 7 & $\approx 4 \mathrm{~d}$ & $14 \mathrm{~h}, 38 \mathrm{~m}, 34 \mathrm{~s}$ & 5.90 \\
\hline
\end{tabular}

We were able to produce examples of escalatory and non-escalatory cases over $\mathbb{F}_{5}$; this was ascertained by computing the class groups of these particular quadratic function fields and their corresponding dual discriminants. For example, the real quadratic function field of discriminant $D=t^{10}+2 t^{9}+t^{8}+4 t^{7}+2 t^{6}+3 t^{5}+3 t^{4}+4 t^{3}+3 t^{2}+t$ has 3 -rank 2 , but its unusual dual field of discriminant $-3 D$ has 3 -rank 3.

We note that our algorithm in both the imaginary and the unusual case is particularly successful at finding quadratic function fields of high 3-rank and small genus because our method is exhaustive. This means that any examples of a given 3-rank value found by our algorithm are minimal, in the sense that any quadratic field with the same 3rank must have genus at least as large as the examples found by our algorithm. For example, our algorithm beats the Diaz y Diaz method, ([10]; adapted to quadratic function fields in [4]), in the sense that fields of 3-rank equal to 3 were found for genus 4 fields over $\mathbb{F}_{5}$ using our algorithm. The minimal genus yielded by the Diaz y Diaz method in [4] for quadratic function fields over $\mathbb{F}_{5}$ with 3 -rank equal to 3 is $g=5$. For $\mathbb{F}_{11}, \mathbb{F}_{13}$ and 3 -rank values 3 and 4 , we also found examples of smaller genus than those given in [4]. The minimal genus values were the same for $\mathbb{F}_{7}$ and 3-rank 4 for both methods. 
TABLE 6. Minimal genera: Our method versus Diaz y Diaz's method ( $-3 D$ imaginary)

\begin{tabular}{|c|c|c|c|r|}
\hline$q$ & 3 -rank & $\min g(\mathrm{DyD})$ & $\min g$ (RJS) & Example $D(t)$ \\
\hline 5 & 3 & 5 & 4 & $t^{9}+2 t^{6}+2 t^{3}+3$ \\
\hline 7 & 3 & 4 & 3 & $3 t^{7}+4 t^{6}+t^{2}+5 t+1$ \\
\cline { 2 - 5 } & 4 & 4 & 4 & $3 t^{9}+5 t^{8}+2 t^{7}+$ \\
& & & & $2 t^{4}+3 t^{3}+5 t^{2}+t+1$ \\
\hline 11 & 3 & 4 & 3 & $2 t^{7}+3 t^{6}+t^{5}+8 t^{4}+$ \\
& & & & $3 t^{3}+t^{2}+3 t+8$ \\
\hline 13 & 3 & 4 & 2 & $t^{5}+10 t^{3}+8 t^{2}+1$ \\
\cline { 2 - 5 } & 4 & 4 & 3 & $t^{7}+10 t^{6}+12 t^{5}+5 t^{4}+$ \\
& & & & $3 t^{3}+11 t^{2}+4 t+10$ \\
\hline
\end{tabular}

TABLE 7. Minimal genera: Our method versus Diaz y Diaz's method ( $-3 D$ unusual)

\begin{tabular}{|c|c|c|c|r|}
\hline$q$ & 3-rank & $\min g$ (DyD) & $\min g$ (RJS) & Example $D(t)$ \\
\hline 5 & 3 & 5 & 4 & $2 t^{10}+4 t^{9}+2 t^{8}+3 t^{7}+$ \\
& & & & $4 t^{6}+t^{5}+t^{4}+3 t^{3}+$ \\
& & & & $t^{2}+2 t$ \\
\hline 7 & 3 & 4 & 3 & $3 t^{8}+t^{6}+5 t^{2}+4 t+6$ \\
\hline
\end{tabular}

An explicit comparison of our method to Diaz y Diaz's algorithm is given in Tables 6 and 7. The third column denotes the minimal genus found with Diaz y Diaz's method yielding the corresponding 3-rank specified in column 2. The fourth column denotes the minimal genus found with our method for the same 3-rank. The fifth column gives an example discriminant of minimal degree with given 3-rank found by our method. Table 7 treats the case where $-3 D$ is unusual. These tables indicate a genuine improvement over previous methods in this regard with respect to finding minimal genera with high 3-rank.

\section{Observations on our numerical data.}

8.1. Divisibility properties of the field counts. We note that various values in Table 3 have some interesting divisibility properties. For instance, all entries in column 5 are divisible by $q$, entries in column 5 for odd degree discriminants are divisible by 2 , and some 
entries in column 5 are divisible by $q-1$. These properties can be explained via automorphisms in the field $\mathbb{F}_{q}(t)$. We now briefly discuss the effect of the $\mathbb{F}_{q}(t)$-automorphisms on the number of discriminants in the various columns of Table 3, thereby solving an open question from $[\mathbf{2 7}, \mathbf{2 8}]$. We note that the counts in column 3 are always of the form $k(q-1) q^{d-1}$ where $d=\operatorname{deg}(d)$ is the discriminant degree and $k=2$ for $d$ odd and $k=1$ for $d$ even. A standard inclusion-exclusion argument proves that the number of monic square-free polynomials of degree $d \in \mathbb{N}$ with coefficients in $\mathbb{F}_{q}$ is $(q-1) q^{d-1}$; see, for example, [21, Exercise 2, subsection 4.6.2, page 456, and its solution on page 679]. The value of $k$ is also easily explained: for odd degree, there are two choices for $\operatorname{sgn}(D)$, namely, 1 and $h$, whereas for $d$ even, there is one, namely $h$. Note also that every quadratic function field over $\mathbb{F}_{q}$ is the function field of a curve of the form $y^{2}=D(t)$ where $D(t)$ is a fundamental discriminant. Our first result explains why the 3-rank totals in column 5 of Table 3 are divisible by $q$.

Proposition 8.1. Assume that $d \geq 3$ and $q$ does not divide $d$. Then, for any 3-rank $r$, the number of imaginary/unusual/real fundamental discriminants of degree $d$ that define a quadratic function field of 3-rank $r$ is a multiple of $q$.

Proof. Let $D(t) \in \mathbb{F}_{q}[t]$ be a fundamental discriminant of degree $d$. Then the polynomials $D(t+\beta)$ with $\beta \in \mathbb{F}_{q}$ are all fundamental discriminants of the same type (imaginary/unusual/real). Since $q \nmid d$, $D(t)$ cannot be a polynomial in $t^{q}-t$, by Lemma 4.1 , the polynomials $D(t+\beta)$ with $\beta \in \mathbb{F}_{q}$ are pairwise distinct. Furthermore, the $q$ curves $y^{2}=D(t+\beta)$ are all isomorphic and hence define the same quadratic function field.

It remains to explain why the entries of column 5 for which the discriminant degree is a multiple of $q$ are also divisible by $q$. Note that the proof of Proposition 8.1 remains valid for fundamental discriminants $D$ such that $q \mid \operatorname{deg}(D)$ but $D$ is not a polynomial in $t^{q}-t$. For the remaining discriminants, the divisibility by $q$ is simply an artifact of the small parameter sizes and no longer holds for larger values of $q$ and $\operatorname{deg}(D)$. For example, the quadratic function field $\mathbb{F}_{q}\left(t, \sqrt{t^{q}-t}\right)$ has 3 -rank 3 for $q=11$ and 3-rank 6 for $q=13$. Explicit formulas for the 
class numbers of $\mathbb{F}_{q}\left(t, \sqrt{t^{q}-t+j}\right)$ with $q$ an odd prime can be found in [11].

Next, we explain why each entry in column 5 of Table 3 is even.

Proposition 8.2. Assume that $q$ is odd, and let $d \geq 3$ be odd. Then, for any 3-rank $r$, the number of imaginary fundamental discriminants of degree $d$ that define a function field of 3-rank $r$ is even.

Proof. Suppose $D(t)$ is monic. Then the curves

$$
y^{2}=D(t) \quad \text { and } \quad\left(h^{(d-1) / 2} y\right)^{2}=D(h t)
$$

are isomorphic over $\mathbb{F}_{q}[t]$ and hence have the same quadratic function field. The result now follows since the distinct polynomials $D(t)$ and $h^{1-d} D(h t)$ are imaginary fundamental discriminants with respective leading coefficients 1 and $h$.

We remark that many, but not all, of the entries in column 5 of Table 3 are divisible by $q-1$. This is likely due to the fact that, most of the time, for the corresponding discriminants $D(t)$, the polynomials $D($ at $)$ with $a \in \mathbb{F}_{q}^{*}$ are pairwise distinct. For example, we have the following result:

Proposition 8.3. Assume that $q$ is an odd prime and $D(t) \in \mathbb{F}_{q}[t]$ is a fundamental discriminant with $D(t)$ monic when $\operatorname{deg}(D)$ is odd. Suppose that, for at least one $j \in\{0,1, \ldots d-1\}$ with $d-j$ coprime to $q-1$, the coefficient of $t^{j}$ in $D(t)$ is non-zero. Then the $q-1$ hyperelliptic curves $y^{2}=h^{-2\lfloor i d / 2\rfloor} D\left(h^{i} t\right)$ for $0 \leq i \leq q-2$ are pairwise distinct and isomorphic and hence have the same quadratic function field.

Proof. For brevity, set $F_{i}(t)=h^{-2\lfloor i d / 2\rfloor} D\left(h^{i} t\right)$. If $d$ is even, then all the $F_{i}(t)$ have the same leading coefficient, namely, $\operatorname{sgn}(D)$. So they are either all real or all imaginary fundamental discriminants. If $d$ is odd, then $F_{i}(t)$ is monic if $i$ is even and has leading coefficient $h$ if $i$ is odd; in either case, all $F_{i}(t)$ are imaginary fundamental discriminants. Hence, the hyperelliptic curves $y^{2}=F_{i}(t)$ are well defined and isomorphic over $\mathbb{F}_{q}$, and therefore they all have the same function field. 
It remains to show that the polynomials $F_{i}(t)$ are pairwise distinct. If $a_{j}$ is the coefficient of $t^{j}$ in $D(t)$, then the coefficient of $t^{j}$ in $F_{i}(t)$ is $a_{j} h^{i j-2\lfloor i d / 2\rfloor}$. So assume that $a_{j} \neq 0$ and $\operatorname{gcd}(q-1, d-j)=1$, and suppose that $F_{i}(t)=F_{k}(t)$ with $0 \leq i, k \leq q-2$. Then

$$
h^{i j-2\lfloor i d / 2\rfloor}=h^{k j-2\lfloor k d / 2\rfloor} .
$$

If $d$ is even, then (8.1) reduces to $h^{(j-d)(i-k)}=1$, so $(j-d)(i-k) \equiv 0$ $(\bmod q-1)$. Since $j-d$ is coprime to $q-1$, this forces $i=k$.

If $d$ is odd, then $i$ and $k$ must be of the same parity, as otherwise one of $F_{i}(t)$ would be monic and the other would have sign equal to $h$. The exact same argument as for $d$ even again forces $i=k$.

Most discriminants satisfy the condition of Proposition 8.3. If those discriminants that do not satisfy this condition correspond to function fields of 3 -rank 0 over $\mathbb{F}_{q}$, then the number of fields with positive 3 rank is divisible by $q-1$ for a given $D$. Note, however, that this is not always the case; counterexamples include $q=5, \operatorname{deg}(D) \in\{5,8\}$ and $q=7, \operatorname{deg}(D) \in\{3,6\}$ (see column "Total" of Tables 3 and 4 ).

8.2. Comparison of 3-rank data to heuristics. Since our algorithm is exhaustive for imaginary or unusual discriminants $-3 D$ of fixed degree, we compared our data to the distribution suggested by the Friedman and Washington heuristic (Conjecture 6.1) and the Ellenberg, Venkatesh and Westerland result (Theorem 6.2), in an effort to provide numerical evidence in support of the validity of the results in the case of fixed $q$. A comparison of our 3-rank data for imaginary and unusual discriminants for the field $\mathbb{F}_{5}$ to the expected value predicted by the aforementioned results is given in Figure 1. We compared our data to the value given by (6.2) in the imaginary and unusual cases for $p=3$. The solid line denotes the value of (6.2) for $r=1$, and the dotted line denotes the proportion of 3-rank one values found up to a given bound found by our algorithm. As seen in Figure 1, our data mostly agrees with the predicted asymptotic probability (6.2). The data for $\mathbb{F}_{11}$ (figure omitted) does not agree as closely, but this is likely because computations were not carried out far enough to obtain a sufficient sample size.

We also compared our $\mathbb{F}_{7}$ data to the expression given in (6.2). As expected, the data is a poor fit to this value. As noted in Malle [24], 
Figure 1. Comparison of actual 3-rank data with predicted value (6.1) over $\mathbb{F}_{5}$.

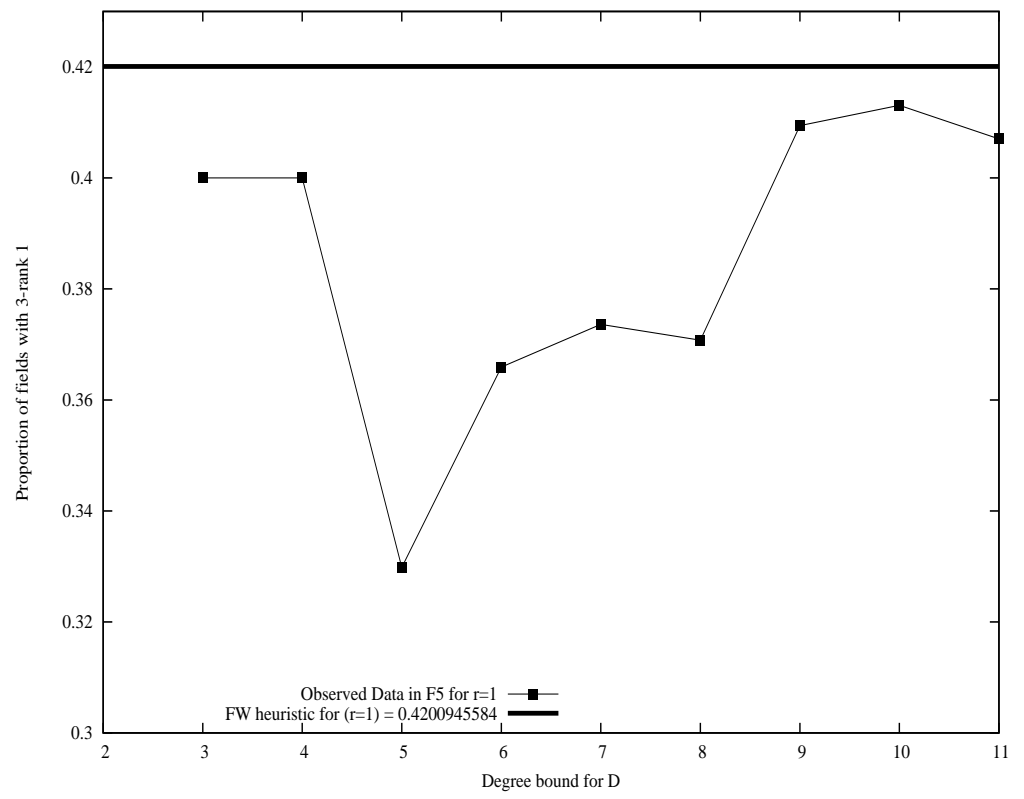

the Cohen, Lenstra and Martinet heuristics for $\ell$-ranks may fail when primitive $\ell$ th roots of unity lie in the base field. This phenomenon occurs for function fields as well, in particular, for the case of $\mathbb{F}_{7}$. For $\ell=3$, we compared our data for $\mathbb{F}_{7}$ to the Garton and Malle formula (6.4) and Achter's $\alpha(g, r)$ function (6.3) in Figure 2. As seen in Figure 2, our data agrees more closely with these predictions. The values of $\alpha(g, 1)$ for $g \leq 3$ appear in [1]. Our data agrees more closely, and in one case exactly, with this formula.

We conclude by noting that our data also compares favorably to both the Ellenberg et al. and Garton distributions, again giving evidence that the dependence on $q \rightarrow \infty$ could perhaps eventually be removed.

9. Conclusion. We presented extensive data on 3-rank distributions of quadratic function fields of discriminant $D$ with $-3 D$ imaginary or unusual, using our cubic function field tabulation algorithm [28], but 
Figure 2. Comparison of actual 3-rank data with predicted values (6.3) and (6.4) over $\mathbb{F}_{7}$.

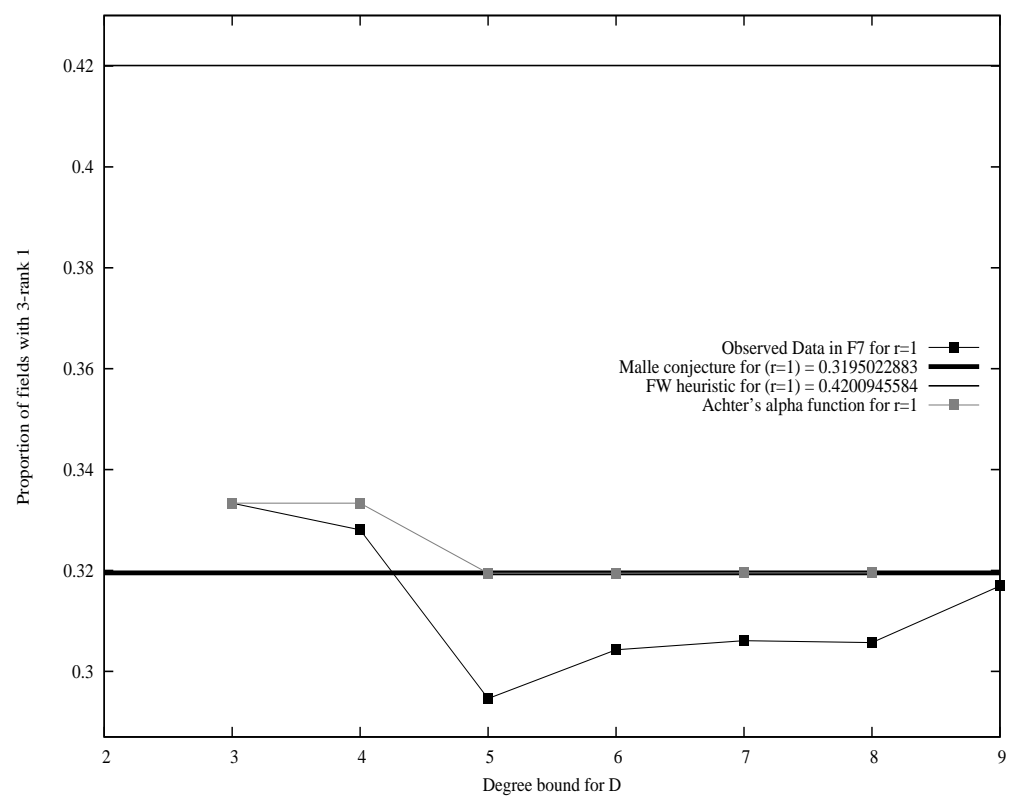

with significant performance improvements in the imaginary case. Our computations were carried out up to degree bounds $\operatorname{deg}(D) \leq 13$ for $q=5$ and various smaller bounds for $q=7,11,13$. In addition, we were able to give an improved algorithm in the imaginary case, which allowed us to extend our computations further than those in [27]. Finally, we accumulated enough data to allow for a meaningful comparison of our 3-rank data with the Friedman-Washington heuristics [14], along with the results of Ellenberg et al. [12], Malle [24, 25] and Garton [17, 16]. Our results approach these heuristics as $\operatorname{deg}(D)$ grows.

It would be interesting to modify the algorithm to the case where $-3 D$ is real. As noted in $[\mathbf{2 7}, \mathbf{2 8}]$, this is currently an open problem. In this case, it is unclear how to efficiently single out a unique reduced representative in each equivalence class of binary cubic forms, and in fact, there are generally exponentially many reduced forms in a given equivalence class. 
We were unable to make use of $\mathbb{F}_{q}(t)$-automorphisms for the case where $-3 D$ is unusual. This is mainly due to the fact that, in this case, translation of a reduced form by $\beta$ results in a partially reduced form and not a reduced form. Losing this unique form in the first part of the modified algorithm means a different approach must be used in order to maintain accurate discriminant counts. One could attempt to just use partially reduced forms instead, but a method to avoid double-counting is needed. Storing all the forms found so far in memory would be one solution but would result in a significantly slower algorithm with more overhead than the original algorithm. Trying to find specific symbolic transformation matrices to get from a type 1 form to a reduced form also seems to be a challenge. In fact, it is unknown to the authors how many type 1, 2 or 3 forms lie in a given cycle of partially reduced unusual forms. Getting this or any speed up to work in the unusual case is currently an open problem.

Computing $\ell$-ranks for quadratic function fields where $\ell \neq 3$ via a similar indirect technique would also be of interest. This requires a special connection between certain higher degree function fields and $\ell$ rank values of quadratic function fields, as given by Hasse in the case of cubic fields and $\ell=3$. Such a connection, along with a tabulation algorithm, was provided in [33] for odd primes $\ell$ dividing $q-1$ and degree $\ell$ function fields whose Galois closure has Galois group $\mathcal{D}_{\ell}$, the dihedral group of $2 \ell$ elements. As yet, the tabulation method of [33] has not been directly applied to the problem of finding quadratic function fields of high $\ell$-rank, and it is unclear how efficient it would be at performing this task. Other techniques for computing $\ell$-ranks of quadratic function fields are currently being investigated. Some recent work has been done on generating data and developing a heuristic for the case where the prime $\ell$ for which the $\ell$-rank is under consideration is equal to the characteristic of the field. See [7] for some new results along these lines.

\section{REFERENCES}

1. Jeffrey D. Achter, The distribution of class groups of function fields, J. Pure Appl. Alg. 204 (2006), 316-333.

2. Math. 463, American Mathermatical Society, Providence, RI, 2008.

3. Emil Artin, Quadratische Körper im Gebiete der höheren Kongruenzen I, Math. Z. 19 (1924), 153-206. 
4. Mark Bauer, Michael J. Jacobson, Jr., Yoonjin Lee and Renate Scheidler, Construction of hyperelliptic function fields of high three-rank, Math. Comp. $\mathbf{7 7}$ (2008), 503-530.

5. Karim Belabas, On quadratic fields with large 3-rank, Math. Comp. 73 (2004), 2061-2074.

6. Lisa Berger, Jing-Long Hoelscher, Yoonjin Lee, Jennifer Paulhus and Renate Scheidler, The l-rank structure of a global function field, Fields Inst. Comm. 60, American Mathematical Society, 2011.

7. Bryden Cais, Jordan S. Ellenberg and David Zureick-Brown, Random Dieudonné modules, random p-divisible groups, and random curves over finite fields, J. Inst. Math. Jussieu 12 (2013), 651-676.

8. Henri Cohen and Hendrik W. Lenstra, Jr., Heuristics on class groups, Lect. Notes Math. 1052, Springer, Berlin, 1984.

9. _ Heuristics on class groups of number fields, Lect. Notes Math. 1068, Springer, Berlin, 1984.

10. Francisco Diaz y Diaz, On some families of imaginary quadratic fields, Math. Comp. 32 (1978), 637-650.

11. Iwan Duursma, Class numbers for some hyperelliptic curves, de Gruyter, Berlin, 1996.

12. Jordan Ellenberg, Akshay Venkatesh and Craig Westerland, Homological stability for Hurwitz spaces and the Cohen-Lenstra conjecture over function fields, available at http://arxiv.org/abs/0912.0325, 2009.

13. Ke Qin Feng and Shu Ling Sun, On class number of quadratic function fields, World Scientific Publishers, Teaneck, NJ, 1990.

14. Eduardo Friedman and Lawrence C. Washington, On the distribution of divisor class groups of curves over a finite field, de Gruyter, Berlin, 1989.

15. Christian Friesen, Class group frequencies of real quadratic function fields: the degree 4 case, Math. Comp. 69 (2000), 1213-1228.

16. D. Garton, Random matrices, the Cohen-Lenstra heuristics, and roots of unity, Alg. Num. Theor. 9 (2015), 149-171.

17. Derek Garton, private communication, 2010.

tics for global fields with roots of unity, 2012 andom atrilable at http://gradworks . umi.com/35/21/3521978.html.

18. Helmut Hasse, Arithmetische Theorie der kubischen Zahlkörper auf klassenkörpertheoretischer Grundlage, Math. Z. 31 (1930), 565-582.

19. M.J. Jacobson, Jr., Y. Lee, R. Scheidler and H.C. Williams, Construction of all cubic function fields of a given square-free discriminant, Inter. J. Num. Theor. 11 (2015), 1839-1885.

20. Michael J. Jacobson, Jr., Shantha Ramachandran and Hugh C. Williams, Numerical results on class groups of imaginary quadratic fields, Lect. Notes Comp. Sci. 4076, Springer, Berlin, 2006. 
21. Donald E. Knuth, The art of computer programming, Volume 2, third Addison-Wesley Publishing Co., Reading, Mass., 1998.

22. Yoonjin Lee, Cohen-Lenstra heuristics and the Spiegelungssatz; function fields, J. Num. Theor. 106 (2004), 187-199.

23. , The Scholz theorem in function fields, J. Num. Theor. 122 (2007), 408-414.

24. Gunter Malle, Cohen-Lenstra heuristic and roots of unity, J. Num. Theor. 128 (2008), 2823-2835.

25. On the distribution of class groups of number fields, Exper. Math. 19 (2010), 465-474.

26. Michael E. Pohst, On computing non-Galois cubic global function fields of prescribed discriminant in characteristic > 3, Publ. Math. Debr. 79 (2011), 611621.

27. Pieter Rozenhart, Fast tabulation of cubic function fields, Ph.D. thesis, University of Calgary, 2009.

28. Pieter Rozenhart, Michael J. Jacobson, Jr. and Renate Scheidler, Tabulation of cubic function fields via polynomial binary cubic forms, Math. Comp. 81 (2012), 2335-2359.

29. Pieter Rozenhart and Renate Scheidler, Tabulation of cubic function fields with imaginary and unusual Hessian, Lect. Notes Comp. Sci. 5011, Springer, 2008.

30. Victor Shoup, Number theory library (NTL), version 5.5.2, 2010, http: //www. shoup.net/ntl.

31. Herman te Riele and Hugh Williams, New computations concerning the Cohen-Lenstra heuristics, Exper. Math. 12 (2003), 99-113.

32. Akshay Venkatesh and Jordan S. Ellenberg, Statistics of number fields and function fields, Volume II, Hindustan Book Agency, New Delhi, 2010.

33. Colin Weir, Renate Scheidler and Everett W. Howe, Constructing and tabulating dihedral function fields, Mathematical Science Publishers, Berkeley, CA, 2013.

Department of Mathematics and Statistics, University of Calgary, 2500 University Drive NW, Calgary, Alberta, Canada T2N 1N4

Email address: pmrozenh@alumni.uwaterloo.ca

Department of Computer Science, University of Calgary, 2500 University Drive NW, Calgary, Alberta, Canada T2N 1N4

Email address: jacobs@cpsc.ucalgary.ca

Department of Mathematics and Statistics, University of Calgary, 2500 University Drive NW, Calgary, Alberta, Canada T2N 1N4

Email address: rscheidl@ucalgary.ca 